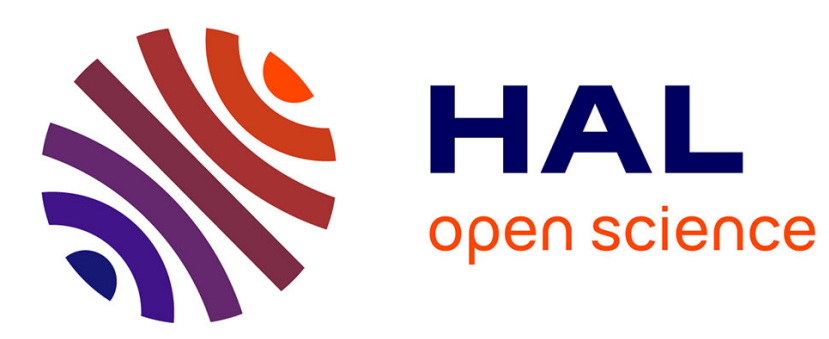

\title{
Optimization of a Synthetic Jet Actuator for Aerodynamic Stall Control
}

Régis Duvigneau, Michel Visonneau

\section{To cite this version:}

Régis Duvigneau, Michel Visonneau. Optimization of a Synthetic Jet Actuator for Aerodynamic Stall Control. Computers and Fluids, 2006, 35 (6), pp.624-638. hal-01730875

\section{HAL Id: hal-01730875 \\ https://hal.inria.fr/hal-01730875}

Submitted on 14 Mar 2018

HAL is a multi-disciplinary open access archive for the deposit and dissemination of scientific research documents, whether they are published or not. The documents may come from teaching and research institutions in France or abroad, or from public or private research centers.
L'archive ouverte pluridisciplinaire HAL, est destinée au dépôt et à la diffusion de documents scientifiques de niveau recherche, publiés ou non, émanant des établissements d'enseignement et de recherche français ou étrangers, des laboratoires publics ou privés. 


\title{
Optimization of a Synthetic Jet Actuator for Aerodynamic Stall Control
}

\author{
Régis Duvigneau and Michel Visonneau* \\ Laboratoire de Mécanique des Fluides CNRS UMR 6598 \\ Ecole Centrale de Nantes \\ B.P. 92101, rue de la Noe FR-44321 Nantes, France \\ Michel.Visonneau@ec-nantes.fr
}

\begin{abstract}
The numerical simulation of aerodynamic stall control using a synthetic jet actuator is presented and the automatic optimization of the control parameters is investigated. Unsteady Reynolds-averaged NavierStokes equations are solved on unstructured grids using a near-wall low-Reynolds number turbulence closure to simulate the effects of a synthetic jet, located at $12 \%$ of the chord from the leading edge of a NACA 0015 airfoil, for a Reynolds number $R e=8.9610^{5}$ and incidences between 12 to 24 degrees. Then, an automatic optimization procedure coupled with the flow solver is employed to optimize the parameters of the actuator (momentum coefficient, frequency, angle with respect to the wall) at each incidence in order to increase the time-averaged lift. A significant increase of the maximum lift is obtained $(+52 \%$ with respect to the baseline airfoil) and the stall delayed from $16^{\circ}$ to $22^{\circ}$ for optimal parameters. The flow characteristics and the influence of the respective control parameters are analysed.
\end{abstract}

Keywords : Flow control, Navier-Stokes, optimization, aerodynamic stall, synthetic jet 


\section{Nomenclature}

\begin{tabular}{|c|c|}
\hline$L$ & airfoil chord \\
\hline$L_{T E}$ & distance between the trailing edge and jet location \\
\hline$U_{\infty}$ & free-stream velocity \\
\hline$\nu$ & kinematic viscosity \\
\hline$R e=U_{\infty} L / \nu$ & Reynolds number \\
\hline$\vec{d}_{j e t}$ & jet direction \\
\hline$\alpha_{\text {jet }}$ & angle of the jet w.r.t. the wall \\
\hline$U_{\text {exp }}$ & velocity amplitude of the jet \\
\hline$U_{\text {jet }}=U_{\text {exp }} / U_{\infty}$ & non-dimensional jet velocity amplitude \\
\hline$N_{e x p}$ & frequency of the jet \\
\hline$N_{j e t}=N_{\text {exp }} L / U_{\infty}$ & non-dimensional jet frequency \\
\hline$N_{j e t}^{\star}=N_{e x p} L_{T E} / U_{\infty}$ & modified non-dimensional jet frequency \\
\hline$h$ & actuation surface \\
\hline$C_{\mu}=h \operatorname{Sin}\left(\alpha_{j e t}\right) U_{\text {exp }}^{2} / L U_{\infty}^{2}$ & blowing coefficient \\
\hline$t_{\exp }$ & physical time \\
\hline$t=t_{\exp } U_{\infty} / L$ & non-dimensional time \\
\hline$\Delta t$ & non-dimensional time step \\
\hline$\overline{U_{\text {jet }}^{\text {opt }}}$ & mean optimal velocity amplitude \\
\hline$\overline{\alpha_{\text {jet }}^{o p t}}$ & mean optimal angle \\
\hline$\overline{N_{j e t}^{o p t}}$ & mean optimal frequency \\
\hline$F$ & cost function \\
\hline$D$ & design variables \\
\hline$Q$ & flow variables \\
\hline$L_{i} \quad L_{s}$ & bound constraints \\
\hline
\end{tabular}

\section{Introduction}

Flow control applied to airfoils has been an active topic of research for many years. Indeed, the characteristics of an airfoil, such as lift, drag or pitching moment, may be adjusted using flow control strategies, without modification of the angle of attack or flap deflection. Therefore, active flow control may produce interesting approaches for a large variety of problems, e.g. changing lift for rotary wing aircraft[1], designing minimum radar cross-section aircraft or delaying aerodynamic stall to enhance maximum lift[2].

However, most of the flow control techniques based on steady jet suction or blowing are difficult to implement into airfoils in practice, since they require large amount of power and room for air supply. Recently, an innovative actuator, called synthetic jet and based on high frequency zero-net-mass-flux injection, was tested experimentally $[3,4,5]$. This new actuator only requires electrical power and may be easily implemented in practical airfoils. Its capability to increase the lift for a cylinder in cross flow was demonstrated[6], whereas post-stall lift increase for symmetric airfoils was also reported[3]. The control parameters, such as the frequency actuation, the location of the blowing slot and the momentum coefficient were also investigated $[3,2]$.

Several numerical investigations concerning lift increase using synthetic jets are reported in the litterature, solving time-accurate Reynolds-Averaged Navier-Stokes Equations (RANS). Large Eddy Simulations (LES) computations at high Reynolds number $\left(R e \approx 10^{6}\right)$ are still too expensive to study such phenomena. Wu et al. [7, 8] investigated post-stall lift enhancement for the NACA 0012 airfoil using suction/blowing normal to the surface located at $2.5 \%$ of the chord and found that lift increase in the post-stall regime can be achieved, as was reported in experiments. The same approach was employed by Hassan et al.[1] for a slot at $13 \%$ of the chord, for different amplitudes and frequencies. It was reported that a high momentum coefficient was required to obtain a significant lift increase. Steady blowing as 
well as oscillatory jet actuations were simulated by Donovan et al. [9] and compared to experimental measurements performed by Seifert et al.[2]. The same configuration was also studied by Ekaterinaris[10], who tested some different jet parameters.

Numerical simulations of flow control are interesting to obtain an accurate analysis of the phenomena involved and it was shown in the works cited above that flow control physics is quite well reproduced by flow solvers. Numerical simulations are also aimed at optimizing the parameters of the actuation, but a systematic search "by hand" of the optimal values is time consuming, since one time-accurate computation has to be performed for each attempt. Therefore, the automatic optimization of the parameters of a synthetic jet (momentum coefficient, frequency and angle with respect to the wall) is investigated in the present study, by coupling an optimization algorithm with the flow solver.

Incompressible Reynolds-averaged Navier-Stokes equations are solved on unstructured grids using the near-wall low-Reynolds number SST $k-\omega$ model of Menter[11] in order to compute the lift of the NACA 0015 airfoil, for a Reynolds number $R e=8.9610^{5}$. The actuation process is a synthetic jet located at $12 \%$ of the chord, which is represented by prescribed velocity boundary conditions. The parameters of the synthetic jet, ie momentum coefficient, frequency and the direction of the outlet, are automatically optimized thanks to an optimization procedure included in the flow solver and already validated by the authors for shape optimization purpose[12]. It is based on the multi-directional search algorithm of Torczon $[13,14]$. This method is a derivative-free approach and is easier to implement in sophisticated flow solvers than gradient-based methods. Moreover, it is less sensitive than gradient-based methods to the numerical noise arising from the numerical process as soon as complex flows are considered. The resulting optimization procedure coupled with the flow solver is employed to search the optimal parameters of the synthetic jet, for a incidence varying from $12^{\circ}$ to $24^{\circ}$.

\section{Flow solver}

The ISIS flow solver, developed by DMN (Division Modélisation Numérique i.e. CFD Department of the Fluid Mechanics Laboratory), uses the incompressible Unsteady Reynolds-averaged Navier Stokes equations (URANS). The solver is based on the finite volume method to build the spatial discretization of the transport equations. The face-based method is generalized to two-dimensional, rotationally-symmetric, or three-dimensional unstructured meshes for which non-overlapping control volumes are bounded by an arbitrary number of constitutive faces. The velocity field is obtained from the momentum conservation equations and the pressure field is extracted from the mass conservation constraint, or continuity equation, transformed into a pressure-equation. In the case of turbulent flows, additional transport equations for modeled variables are solved in a form similar to the momentum equations and they can be discretized and solved using the same principles.

\subsection{Conservation equations}

For an incompressible flow of viscous fluid under isothermal conditions, mass, momentum and volume fraction conservation equations can be written as (using the generalized form of Gauss' theorem):

$$
\begin{aligned}
& \frac{\partial}{\partial t} \int_{V} \rho d V+\int_{S} \rho\left(\vec{U}-\vec{U}_{d}\right) \cdot \vec{n} d S=0 \\
& \frac{\partial}{\partial t} \int_{V} \rho U_{i} d V+\int_{S} \rho U_{i}\left(\vec{U}-\vec{U}_{d}\right) \cdot \vec{n} d S \\
& \quad=\int_{S}\left(\tau_{i j} I_{j}-p I_{i}\right) \cdot \vec{n} d S+\int_{V} \rho g_{i} d V
\end{aligned}
$$

where $V$ is the domain of interest, or control volume, bounded by the closed surface $S$ moving at the velocity $\vec{U}_{d}$ with a unit normal vector $\vec{n}$ directed outward. $\vec{U}$ and $p$ represent respectively the velocity 
and pressure fields. $\tau_{i j}$ and $g_{i}$ are the components of the viscous stress tensor and the gravity, whereas $I_{j}$ is a vector whose components vanished, except for the component $j$ which is equal to unity.

\subsection{Turbulence modeling}

Several turbulence closures are included in the flow solver, ranging from linear eddy-viscosity based models to full second order closures [15]. For these studies, the near-wall low-Reynolds SST $k-\omega$ model of Menter[11] is chosen, since it has proved to behave satisfactorily for separated flows over airfoils.

\subsection{Numerical framework}

\subsubsection{Spatial discretization}

All the flow variables are stored at geometric centers of the arbitrary shaped cells. Surface and volume integrals are evaluated according to second-order accurate approximations by using the values of integrand that prevail at the center of the face $f$, or cell $C$, and neighbor cells. The various fluxes appearing in the discretized equations are built using centered and/or upwind schemes. For example, the convective fluxes are obtained by two kinds of upwind schemes. A first scheme available in the flow solver, (HD) for Hybrid differencing, is a combination of upwind (UD) and centered (CD) schemes. Contrary to a practical approach $[16,17]$ where $\mathrm{CD} / \mathrm{UD}$ blending is fixed with a global blending factor for all faces of the mesh, the HD scheme results from a local blending factor based on the signed Peclet number at the face. An other upwind scheme which is implemented in ISIS, is the Gamma Differencing Scheme (GDS) [18]. Through a normalized variable diagram (NVD) analysis [19], this scheme enforces local monotonicity and convection boundedness criterium (CBC) [20]. A pressure equation is obtained in the spirit of the Rhie and Chow [21] procedure and momentum and pressure equations are solved in an segregated way like in the well-known SIMPLE coupling procedure.

\subsubsection{Temporal discretization}

The temporal discretization of a variable $A$ is based on a three-step upwind discretization :

$$
\frac{\partial A}{\partial t} \approx \frac{\delta A}{\delta t}=e^{c} A^{c}+e^{p} A^{p}+e^{q} A^{q}
$$

the index $c$ corresponding to the current time step and the indices $p$ et $q$ to the past time steps. The coefficients $e^{c}, e^{p}$ and $e^{q}$ are chosen to ensure a second-order accuracy. The convection and diffusion are treated implicitly.

\subsubsection{Boundary conditions}

No slip conditions are imposed on the walls. An imposed velocity field is used on the far-field boundary. Concerning the synthetic jet actuator, a suction/blowing type boundary condition is used, imposing a prescribed velocity :

$$
\vec{U}=U_{\text {jet }} \sin \left(2 \pi N_{\text {jet }} t\right) f(s) \vec{d}_{\text {jet }}
$$

where $\vec{d}_{\text {jet }}$ is a vector of unit length representing the direction of the jet outlet, $\alpha_{j e t}$ being the angle between $\vec{d}_{j e t}$ and the wall. $f(s)$ is the tangential distribution of the velocity. The spatial variation of the jet in the tangential direction is supposed to have a negligible influence on the flow, as shown by Donovan et al.[9]. Therefore, a "top hat" tangential distribution is adopted, corresponding to $f(s)=1$. Then, the parameters of the synthetic jet to optimize, called design variables $D$, are respectively the velocity amplitude $U_{j e t}$, the jet frequency $N_{j e t}$ and the direction of the outlet $\alpha_{j e t}$. 


\section{Optimization procedure}

\subsection{Design cycle}

Design optimization consists in maximizing a cost function $F$ depending on the flow variables $Q(D)$ and the design variables $D$. The governing equations of the flow $R(D, Q(D))=0$ are considered as constraints which must be satisfied at each step of the design cycle. Some bound constraints must be added to the problem in order to find a realistic solution. Thus, the variation domain of the design variables is usually closed. From a mathematical point of view, the problem may be expressed as:

$$
\begin{array}{ll}
\text { Maximize } & F(D, Q(D)) \\
\text { Constrained to } & R(D, Q(D))=0 \\
& L_{i} \leq D \leq L_{s}
\end{array}
$$

In the present work, the cost function is the time-averaged lift of the airfoil. Therefore, the design procedure consists in several unsteady simulations of the flow for a synthetic jet with different parameters, or design variables, whose values are modified by an optimization algorithm. Thus, the design cycle may be described by :

(1) Initialization of the design parameters $D$

(2) Unsteady Simulation of the flow $Q(D)$

(3) Evaluation of the time-averaged lift $F(D, Q(D))$

(4) Update of $D$ by the optimization algorithm

(5) Goto step (2)

until the convergence of the design variables is achieved.

\subsection{Optimization algorithm}

\subsubsection{Strategy}

The optimizer plays a crucial role in the design procedure, since it predicts improved design variables at each optimization step, from the information collected previously. The use of gradient-based optimizers is usually motivated by their efficiency, since they can reach a minimum of the cost function in a number of evaluations lower than zero-order methods. However, some difficulties arise when they are faced with complicated realistic problems. The evaluation of the derivatives of the cost function for a sophisticated simulation process is rather problematic. Their evaluation is usually based on an adjoint formulation, relying on the differentiation of the flow solver [22,23]. This task is tedious when high-order discretization schemes on unstructured grids are used, or complex turbulence models are employed. This approach is often implemented with simplifications of the problem, neglecting turbulence sensitivities for instance, or using first-order discretization schemes, which provides an approximated gradient. It has been shown by Anderson and Nielsen [24] that these simplifications often lead to erroneous gradient values. Thus, this approach is still limited to moderately complicated problems. Moreover, these algorithms are very sensitive to the noisy errors arising from the evaluation of the cost function and generating irregularities and spurious local minima. These sources of errors were studied by Madsen [25], who underlined the highfrequency errors introduced by the use of high-order discretization schemes and low converged solutions.

To overcome these limitations, a derivative-free algorithm, which is easier to implement in a complex numerical framework, is employed. It may be associated with sophisticated flow solvers, since the solver is considered as a black-box, which is not modified when included in the design procedure. Furthermore, this approach is less sensitive to the noise, because no information about the derivatives is needed to predict the optimization path. The number of evaluations required is higher than that necessited by gradient-based methods, but it remains reasonable, as soon as the number of design variables is low, which is the case in the present study. 


\subsubsection{Torczon's algorithm}

The optimization method used to lead the search is based on the multi-directional search algorithm developped by Torczon[13, 14]. This algorithm is inspired from the Nelder-Mead simplex method, used by the authors in the past for shape optimization purpose[12,15]. Torczon proposed modifications to correct some of its drawbacks and to give the possibility to perform parallel computations. Particularly, a proof of convergence is given under classical assumptions concerning the regularity of the cost function[13, 14].

The algorithm used consists in moving a simplex of $n+1$ vertices in $\mathbb{R}^{n}$ (a triangle in $\mathbb{R}^{2}$, a tetrahedron in $\mathbb{R}^{3}$, etc), for a problem of $n$ design parameters, each vertex representing a distinct design. The simplex is first initialized, for instance perturbing the initial design parameters $D$ in each of the $n$ directions independantly. Then, displacements are performed in order to increase at each step the cost function evaluated at the best vertex. At each step $k$, the whole simplex, composed of the vertices $v_{0}^{k}, v_{1}^{k}, \ldots, v_{n}^{k}$ is reflected with respect to the best vertex $v_{0}^{k}$ :

$$
v_{i}^{r}=(1+\alpha) v_{0}^{k}-\alpha v_{i}^{k} \quad i=0, \ldots, n
$$

with $\alpha$ set to unity. In case of success, ie if for all vertices $v_{1}^{r}, \ldots, v_{n}^{r}$ the cost function is higher than $F\left(v_{0}\right)$, the simplex is expanded from $v_{0}^{r}$ :

$$
v_{i}^{e}=\gamma v_{i}^{r}+(1-\gamma) v_{0}^{r} \quad i=0, \ldots, n
$$

with $\gamma$ set to two. On the contrary, in case of failure, ie if for all vertices $v_{1}^{r}, \ldots, v_{n}^{r}$ the cost function is lower than $F\left(v_{0}\right)$, the simplex is contracted from $v_{0}^{r}$ :

$$
v_{i}^{c}=\beta v_{i}^{r}+(1-\beta) v_{0}^{r} \quad i=0, \ldots, n
$$

with $\beta$ set to minus half. If none of the previous cases are observed, ie if some vertices of the reflected simplex are better than $v_{0}^{k}$, the reflected simplex is retained. Then, the algorithm continues with the new simplex for the step $k+1$. The loop is then repeated until a maximum of the cost function is reached.

\section{Application to the NACA 0015 airfoil}

The automatic optimization procedure described above is employed to search the optimal parameters of a synthetic jet. More precisely, the momentum coefficient, the frequency and the angle with respect to the wall are taken into account in the optimization procedure as design variables. A major difficulty in deterministic optimization is the choice of the initial design parameters. Indeed, if their choice is awkward, the optimization procedure may lead to a local maximum yielding a low fitness, because of the non-linearities of the cost function. Moreover, the location of the synthetic jet has to determined. To overcome these difficulties and study a realistic test case, the flow control experiments of Gilarranz et al.[5] around the NACA 0015 airfoil for a Reynolds number $R e=8.9610^{5}$ are considered as starting point for the optimization.

\subsection{Naca 0015 baseline}

Computations for the uncontrolled NACA 0015 airfoil are first performed on different grids, for a Reynolds number $R e=8.9610^{5}$ and incidence angles from $12^{\circ}$ to $24^{\circ}$, in order to assess the numerical accuracy of the calculations. Hybrid meshes are employed, with a multi-block structured grid around the airfoil and in the near-wake, and a triangular grid further where lower gradients are found. The distance between the first node and the wall is $1.010^{-5}$ to fulfill the criterion " $y^{+}<1$ ". Four grids are tested, respectively composed of 32136 cells, 53562 cells, 84577 cells and 149914 cells. Turbulence modeling is achieved by the near-wall SST $k-\omega$ model. The comparison of the lift coefficients computed is shown in figure 1 . As seen, the results are very similar before stall, but differ significantly for the post-stall regime. However, the result obtained for the grids 3 and 4 are close enough to establish that the grid independancy is 
almost reached. One may notice that for all grids, even the coarsest, the stall angle is about $16^{\circ}$. Since a large number of unsteady simulations will be performed in the design procedure, the grid 3 is chosen for the next calculations. For this grid, the number of nodes on the upper face of the airfoil is 364 and a refined area is located at the jet slot, which is described by 45 faces (figure 2).

When these results are compared to the experiments of Gilarranz et al.[5] for the uncontrolled NACA 0015 airfoils, significant differences appear, since stall occurs experimentally at an incidence of about $12^{\circ}$ and the maximum lift coefficient is 1.0 . However, these differences are foreseenable : first, a fully turbulent flow was computed, although transitional effects usually occur at this Reynolds number. Then, the wind tunnel height is only 2.3 chord and therefore blocking effects may arise. Finally, the wind tunnel width is only 3.2 chord and a three-dimensional flow may appears as soon as flow separation takes place, as shown in experimental flow vizualizations[5]. To better fit the experimental measurements, transition as well as wind tunnel walls have to be taken into account in computations, which seems not to be reasonable in a design optimization context because of the computational cost involved. The purpose of the present study is to understand the relations between the parameters of a synthetic jet and their respective influence on the control fitness. Since the experimental datas are only considered as starting parameters for the design optimization, the difficulty of reproducing the experimental conditions and facilities does not seem critical. This hardness to accurately simulate stall was pointed out by several authors $[9,26]$ who studied stall control numerically.

\subsection{Controlled NACA 0015}

In the experiments of Gilarranz et al.[5], the actuation is performed by a synthetic jet located at $12 \%$ of the chord $L$ from the leading edge, with an actuation surface $h=3.05 \%$ of the chord. Because of technical requirements for the actuator, the momentum coefficient is correlated to the frequency of the actuation. This assumption will not be retained for the numerical investigations. Among the values tested experimentally, a non-dimensional frequency $N_{\text {jet }}=N_{\text {exp }} L / U_{\infty}=1.29$ and a non-dimensional velocity amplitude $U_{j e t}=U_{e x p} / U_{\infty}=1.37$ are retained because of the satisfactory results obtained, with $U_{\infty}$ the free stream velocity, $N_{\exp }$ the frequency of the jet and $U_{\exp }$ the velocity amplitude of the jet. The jet outlet is almost tangential to the wall, a small angle $\alpha_{j e t}=10^{\circ}$ being imposed. These values, considered as starting point for the optimization, correspond to a momentum coefficient $C_{\mu}=$ $h \operatorname{Sin}\left(\alpha_{j e t}\right) U_{e x p}^{2} / L U_{\infty}^{2}=0.01$. The use of the non-dimensional frequency proposed by Seifert et al.[2], defined by $N_{\text {jet }}^{\star}=N_{\text {exp }} L_{T E} / U_{\infty}$, with $L_{T E}$ the distance between the trailing edge and the actuation location, leads to a value of $N_{j e t}^{\star}=1.13$, close to the unity value recommended by Seifert et al.[2]. The grid number 3, including 84577 cells is used for the calculations, with the SST $k-\omega$ model. A nondimensional time step $\Delta t=5 \cdot 10^{-3}$ is employed, providing a sufficient temporal resolution to describe the time dependant jet flow. Time-accurate calculations are performed from scratch until the non-dimensional time $t=t_{\exp } U_{\infty} / L=70$, with $t_{\exp }$ the physical time, for which a periodic flow is observed.

The evolution of the lift coefficient with respect to the incidence angle is shown on figure 3 , for the baseline airfoil and the controlled airfoil with initial parameters. As seen, for an incidence of $12^{\circ}$ and $14^{\circ}$ the synthetic jet has no significant influence on the lift coefficient. However, the slope of the curve is maintained until $18^{\circ}$ thanks to the actuation, whereas the slope begins to decrease after $14^{\circ}$ for the baseline airfoil. In the controlled case, the lift decrease occurs at about $19^{\circ}$, delaying the stall by $3^{\circ}$. The maximum lift is increased of $16 \%$. The evolution of the lift coefficient with respect to the non-dimentional time $t$ is shown on figures 4 to 10 , for the final time steps, when a periodic flow is observed. For the baseline airfoil, the flow is rather steady until $18^{\circ}$, which is not the case for the controlled airfoil because of the actuation. The evolution is close to a sinusoidal function with a frequency equal to the frequency actuation, except for an incidence of $24^{\circ}$, where a non-periodic flow is observed for both cases. For this last incidence, the synthetic jet influence on the flow is negligible. 


\subsection{Optimal control parameters}

The automatic optimization of the design parameters $\alpha_{j e t}, U_{j e t}$ and $N_{j e t}$ is then performed for each incidence angle, from the initial parameters described above. The cost function to maximize is the time-averaged lift coefficient $C_{l}$, evaluated during a sufficient long time :

$$
F(D, Q(D))=\frac{1}{t_{2}-t_{1}} \int_{t_{1}}^{t_{2}} C_{l}(D, Q(D)) d t
$$

In practice, the above integration is performed during the last 1000 time steps, 5000 time steps being computed for each design parameters set. Some experiments have shown that these values are large enough to evacuate the transient effects and integrate the lift during several periods. An integration during a fixed number of time steps is preferred to a one-period integration, since it may be tedious in practice to automatically get a precise evaluation of the period of the time dependant lift. When a low frequency actuation is found during the optimization, as seen below, a new optimization exercise is performed using a twice longer integration time, to confirm the results. Between 15 and 20 optimization steps are required in order to stabilize the design parameters at the optimal values found. This corresponds to 45 to 60 unsteady computations for each incidence angle. To reduce the computational time, the flow is computed with a multi-block domain partitioning approach, involving 16 blocks.

The evolution of the cost function during the optimization procedure may be seen in figure 11. For the small incidence angles, the increase of the cost function is rather limited, but progressively increases for higher incidences. One may notice that the optimization procedure for the incidence of $24^{\circ}$ has different initial parameters and initial cost function value. Indeed, the first optimization test using the experimental parameters as initial parameters fails to increase significantly the lift at this incidence. This is due to the presence of a local optimum close to the starting design. Therefore, other initial parameters are chosen, corresponding to the mean optimal values found at other incidences. In this way, a considerably better actuation is found, corresponding to a better optimum. As seen in figure 3, the optimizer fails to significantly increase the lift as long as the flow is fully attached. Nevertheless, when stall occurs for the baseline airfoil, the control with optimal parameters provides a more efficient actuation than the initial control, since the slope of the lift coefficient with respect to the incidence is increased (between $12^{\circ}$ and $16^{\circ}$ ). Moreover, the interval of incidence for which the actuation is efficient is enlarged, since the stall is delayed to $22^{\circ}$, which corresponds to an increase of $52 \%$ of the maximum lift coefficient with respect to the baseline airfoil. This computation clearly shows how critical is the choice of the control parameters to obtain a large stall delay.

The optimal design parameters found are shown on figures 12 to 14 . Except for the incidence $12^{\circ}$, for which no significant progress is observed, some tendancies may be drawn. First, the amplitude of the jet $U_{j e t}$ is increased to a mean value of $\overline{U_{\text {jet }}^{\text {opt }}}=1.72$. As consequence, the lift coefficient amplitude is increased with respect to the initial control (figures 4 to 10). This observation is in accordance with the results of other authors $[9,10,26]$ as well as experimental observations[2]. Then, the angle between the jet outlet and the wall is increased to a a mean value of about $\overline{\alpha_{j e t}^{o p t}}=25^{\circ}$. This evolution is in agreement with the numerical experiments of Ekaterinaris[10] for trailing edge control and Donovan et al.[9] for leading edge control. The most interesting parameter is the frequency. Indeed, except for the incidence $12^{\circ}$, for which no significant progress is observed, five optimal frequencies found are very close to each other, with a mean value of $\overline{N_{\text {jet }}^{\text {opt }}}=0.85$. Only one optimal value, at $22^{\circ}$, significantly differs with very good results, since the optimal frequency found is about $N_{\text {jet }}^{\text {opt } 22}=0.25$. The evolution of the lift with respect to the time shows a tendancy to move away from a simple sinusoidal curve (figures 4 to 10). The amplitude of the oscillations is larger, but the time-averaged lift remains higher than with the initial parameters. This observation is particularly true at $22^{\circ}$, the lift decreasing severely during a short time period at each cycle. The tendancy to increase the oscillations amplitude by reducing the actuation frequency was shown by Ekaterinaris[10]. The figure 15 shows the drag polars for the baseline and both controlled airfoils. As seen, the drag coefficients are very similar as long as the flow is rather attached, until $16^{\circ}$. At $18^{\circ}$ and $20^{\circ}$, the drag coefficient for controlled flows is slightly higher than for the baseline 
airfoil. This may be explained by the fact that no vortex shedding is observed for the baseline airfoil, the flow being rather steady with an increasing recirculation area, contrary to the controlled flow for which a vortex shedding is imposed by the actuation. As soon as the post-stall regime is reached, at $22^{\circ}$, the initial actuation produces a noticeable decrease of the drag, since smaller vortices are generated thanks to the actuation (see flow analysis below). This is not the case for the control with optimal parameters at $22^{\circ}$, since rather large vortices are generated due to the low frequency actuation. Actually, a drag reduction was not aimed during the optimization. At $24^{\circ}$, the initial control has obviously no significant influence on the flow, as seen before. But the control with optimal parameters, with a frequency higher than at $22^{\circ}$, yields to a strong drag reduction. These observations are in accordance with the experimental[2] and numerical $[9,10]$ results found in the litterature.

\subsection{Complementary computations}

Considering the results obtained during the previous optimization exercises, it seems to be interesting to perform some complementary computations to study further the characteristics of the control. Particularly, the capability to obtain a satisfactory control using fixed parameters for all incidences is investigated, since the variations of the optimal paramaters found with respect to the incidence angle look rather complicated. Then, one calculation is performed using the mean optimal values found, ie $\overline{U_{j e t}^{o p t}}=1.72, \overline{\alpha_{j e t}^{o p t}}=25^{\circ}$ and $\overline{N_{j e t}^{o p t}}=0.85$. In that way, one intends to test a configuration, for which the previous tendancies for the amplitude and the angle are verified and the frequency very close to the optimal frequencies found at several incidences $\left(14^{\circ}\right.$ to $20^{\circ}$ and $\left.24^{\circ}\right)$. Moreover, a second test is performed with the optimal parameters found at $22^{\circ}$, ie $U_{\text {jet }}^{\text {opt } 22}=1.87, \alpha_{\text {jet }}^{\text {opt } 22}=35^{\circ}$ and $N_{\text {jet }}^{\text {opt } 22}=0.25$. Therefore, the capapility to perform an efficient control with a low frequency actuation is investigated.

The evolution of the lift coefficient with respect to the incidence angle may be seen in figure 16 . As expected, the new parameters produce an airfoil whose fitness is lower than using optimal parameters. When mean optimal parameters are employed, the lift coefficient increases until $24^{\circ}$, but the maximum lift coefficient is lower than using optimal parameters $(+41 \%$ with respect to the baseline). Moreover, the slope of the lift with respect to the incidence begins to decrease as soon as $20^{\circ}$. Considering the actuation with optimal parameters for $22^{\circ}$, this slope is rather maintained until $22^{\circ}$, providing a control almost as efficient as the control involving optimal parameters at each incidence. However, a stronger lift decrease is observed after $22^{\circ}$. The figures 17 to 23 show the evolution of the lift coefficient with respect to the time. The control with optimal parameters for $22^{\circ}$ generates a lift with larger oscillatory amplitudes at all incidence angles, with a short period of time during which the lift falls abruptly. Nevertheless, the time-averaged lift is higher until $22^{\circ}$ than using a control based on mean optimal values. These large variations of the lift, related to the use of a low frequency actuation, may be a serious drawback in practice, although these design parameters seem to produce the most efficient stall delay, when fixed parameters are used.

\subsection{Flows analysis}

To analyze the influence of the actuation parameters on the flow characteristics, one considers the flows obtained at $22^{\circ}$, for the different parameters mentioned above. Particularly, the time-averaged pressure coefficient on the airfoil is shown on figures 24 and 25, for the baseline airoil, the controlled airfoil with initial parameters, optimal parameters and mean optimal parameters. The velocity streamlines may be seen in figures 26 to 29 . In order to understand the flow dynamics, four times per cycle are represented, which correspond to the actuation at $\Phi=0$ (no mass flow), $\Phi=\pi / 2$ (maximum blowing), $\Phi=\pi$ (no mass flow) and $\Phi=3 \pi / 2$ (maximum suction), and to the natural shedding for the baseline airfoil.

\subsection{1 baseline}

For the NACA 0015 airfoil without actuation, a large region of separated flow is observed at the upper face of the airfoil for all times (figure 26). The lift variations are due to the development and shedding 
of two counter-rotative vortices. As consequence, the time-averaged pressure coefficient is low for $90 \%$ of the upper face of the airfoil (figure 24), providing low suction effects and a low lift coefficient.

\subsection{2 initial parameters}

For the oscillatory blowing and suction with initial parameters, some (two or three) smaller vortices are observed on the upper face, generated at about $25 \%$ of the chord and convected downstream (figures 27 ). Whatever the time considered, the flow is attached on the first $25 \%$ of the chord, yielding higher suction effects at the leading edge (figure 25). Over the remaining upper face, the flow is rather detached at all time. Therefore, the time variation of the lift is low in this case (figures 9). However, the time-averaged pressure coefficient is significantly higher on the overall airfoil than for the baseline, thanks to the vortices convection, yielding a higher time-averaged lift $(+16 \%$ with respect to the baseline).

\subsubsection{Mean optimal parameters}

Using mean optimal parameters (higher amplitude, higher angle with respect to the wall and lower frequency) a more efficient actuation is observed. As in the previous case, some vortices are generated and convected along the upper face of the airfoil. However, the vortices are clearly separated, the flow being re-attached between them. This characteristic may be related to the use of a lower frequency and higher amplitude actuation. The birth of the vortices takes place at about $20 \%$ of the chord, during the blowing (figure 28, $\Phi=\pi / 2$ ). At the time of maximum lift, at the end of the blowing (between $\Phi=\pi / 2$ and $\Phi=\pi)$, the flow is mainly attached on the first $50 \%$ of the chord and re-attached downwind the vortex. Therefore, the flow is attached on more than half of the upper face of the airfoil. The time-averaged pressure coefficient is higher than for the actuation with initial parameters (figures 24 and $25)$ and the lift is consequently increased ( $+41 \%$ with respect to the baseline). During the suction, the vortices generated are growing $(\Phi=3 \pi / 2)$, and then collapsing as the shedding of the main vortex occurs

$(\Phi=0)$. It corresponds to the minimum lift time. As consequence to the convection of vortices and variation of the flow attached area, the time history of the lift coefficient shows higher variations than for the previous case (figure 22).

\subsubsection{Optimal parameters at $22^{\circ}$}

The optimal parameters at $22^{\circ}$ are mainly characterized by a very low actuation frequency. Therefore, larger vortices appear in the flow. As in the previous case, some small vortices are generated during the blowing $(\Phi=\pi / 2)$, whereas a main vortex is growing during the suction $(\Phi=3 \pi / 2)$. However, As seen in figure 22 , the evolution of the lift with respect to the time is more complex than in the previous case. At the end of the suction (before $\Phi=0$ ), the vortex shedding corresponds to the minimum value of the lift. Then, during the blowing $(\Phi=\pi / 2)$, the flow is mainly attached on the upper face of the airfoil and the lift increases quickly. When the suction occurs $(\Phi=\pi)$, a main vortex is growing as the lift slightly decreases, because of the large separation area. At the maximum suction time $(\Phi=3 \pi / 2)$, the main vortex is convected along the upper face of the airfoil, generating some smaller vortices upstream. Therefore, the lift increases again, since the flow is more attached near the leading edge. Then, between $\Phi=3 \pi / 2$ and $\Phi=0$ the main vortex is located near the trailing edge. Therefore, the flow is attached on about $70 \%$ of the upper face of the airfoil. As consequence, higher suction effects are provided (figures 24 and 25), which corresponds to a very high instantaneous lift. However, the lift then falls abruptly as the vortex shedding occurs, as explained above. Finally, a highest time-averaged lift $(+52 \%$ with respect to the baseline) is observed. Nevertheless, the variation of the lift with respect to the time is particularly large. 


\section{Conclusion}

The simulation of aerodynamic stall control using a synthetic jet actuator is performed, solving unsteady Reynolds-averaged Navier-Stokes equations, for a NACA 0015 airfoil at Reynolds number $R e=8.9610^{5}$. The flow solver is then coupled with an automatic optimization procedure, which relies on the derivativefree multi-directional search algorithm of Torczon, to optimize for each incidence the velocity amplitude, the frequency and the angle with respect to the wall, to increase the time-averaged lift.

It is found that the efficiency of the control is significantly improved when optimal parameters are chosen. The maximum lift is increased by $+34 \%$ and the stall delayed from $19^{\circ}$ to $22^{\circ}$, with respect to the initial control parameters. This study clearly shows the interest of coupling an automatic optimization procedure with the flow solver, since the optimal parameters found at each incidence seem not to be easily forseeable and may vary abruptly. However, some tendancies may be drawn : the mean optimal

parameters are, with non-dimensional values, $\overline{U_{\text {jet }}^{o p t}}=1.72, \overline{N_{\text {jet }}^{o p t}}=0.85$ and $\overline{\alpha_{\text {jet }}^{o p t}}=25^{\circ}$. The use of these mean values provides a quite efficient and robust control, which has, however, a significant lower best fitness than the control with optimal parameters for each incidence. Thus, the sensibility of the parameters is underlined.

The flow controlled by the synthetic jet actuator is characterized by the generation of vortices at about $20 \%$ of the chord, which are then convected on the upper face of the airfoil. The decrease of the actuation frequency and the increase of the momentum coefficient yield a higher lift amplitude and larger vortices.The efficiency of the control, ie the increase of the time-averaged lift, seems to be related to the capacity to delay the distachement of the flow and its re-attachement between vortices, on the upper face of the airfoil.

Some additional computations are to be performed to assess these results, providing that no such lift enhancement was reported in the literature. Especially, the influence of turbulence modeling on flow control simulations has to be quantified. Since turbulence closures play a significant role as soon as the flow is detached, one may fear that their influence on flow dynamics and optimal control parameters is not negligible.

\section{Acknowledgements}

The authors gratefully acknowledge the scientific committee of CINES (project dmn2050) and IDRIS (project 1308) for the attribution of CPU time.

\section{References}

[1] Hassan A, Straub F, BD C. Effects of surface blowing/suction on the aerodynamics of helicopter rotor blade-vortex interactions - a numerical simulation. Journal of American Helicopter Society 1997;42:182-194.

[2] Seifert A, Darabi A, Wygnanski I. Delay of airfoil stall by periodic excitation. AIAA Journal 1996; 33(4):691-707.

[3] Seifert A, Bashar T, Koss D, Shepshelovich M, Wygnanski I. Oscillatory blowing : a tool to do delay boundary layer separation. AIAA Journal 1993;31(11):2052-2060.

[4] Smith B, Glezer A. Vectoring and small-scale motions effected in free shear flows using synthetic jet actuators. AIAA Paper 97-0213 1997.

[5] Gilarranz J, Traub L, Rediniotis O. Characterization of a compact, high power synthetic jet actuator for flow separation control. AIAA Paper 2002-0127 2002.

[6] Smith D, Amitay M, Kibens V, Parekh D, Glezer A. Modification of lifting body aerodynamics using synthetic jet actuators. AIAA Paper 98-0209 1998. 
[7] Wu J, Lu X, Denney A, Fan M, Wu J. Post-stall lift enhancement on an airfoil by local unsteady control, part i. lift, drag and pressure characteristics. AIAA paper 97-2063 1997.

[8] Wu J, Lu X, Wu J. Post-stall lift enhancement on an airfoil by local unsteady control, part ii. mode competition and vortex dynamics. AIAA paper 97-2064 1997.

[9] Donovan J, Kral L, Cary A. Active flow control applied to an airfoil. AIAA Paper 98-0210 1998.

[10] Ekaterinaris J. Active flow control of wing separated flow. ASME FEDSM'03 Joint Fluids Engineering Conference, Honolulu, Hawai, USA, July 6-10 2003.

[11] Menter F. Zonal two-equations $k-\omega$ turbulence models for aerodynamic flows. AIAA paper 93-2906 1993.

[12] Duvigneau R, Visonneau M. Shape optimization for incompressible and turbulent flows using the simplex method. AIAA Paper 2001-2533 2001.

[13] Torczon V. Multi-Directional Search: A Direct Search Algorithm for Parallel Machines. Ph.D. thesis, Houston, TX, USA 1989.

[14] Dennis J, Torczon V. Direct search methods on parallel machines. SIAM Journal of Optimization $1991 ; 1(4): 448-474$.

[15] Duvigneau R, Visonneau M, Deng G. On the role played by turbulence closures for hull shape optimization at model and full scale. Journal of Marine Science and Technology 2003;8(1).

[16] Demirdžić I, Muzaferija S. Numerical method for coupled fluid flow, heat transfert and stress analysis using unstructured moving meshes with cells of arbitrary topology. Comput. meth. Appl. Mech. Eng. $1995 ; 125: 235-255$.

[17] Ferziger J, Perić M. Computational methods for fluid dynamics. Springer-Verlag, Berlin 1996.

[18] Jasak H. Error Analysis and Estimation for the Finite Volume Method with Applications to Fluid Flows. Ph.D. thesis, University of London 1996.

[19] Leonard B. Simple high-accuracy resolution program for convective modelling of discontinuities. International Journal for Numerical Methods in Fluids 1988;8:1291-1318.

[20] Gaskell P, Lau A. Curvature ompensated convective transport: SMART , a new boundedness preserving transport algorithme. International Journal for Numerical Methods in Fluids 1988;8:617641.

[21] Rhie C, Chow W. A numerical study of the turbulent flow past an isolated airfoil with trailing edge separation. AIAA Journal 1983;17:1525-1532.

[22] Anderson WK, Venkatakrishnan V. Aerodynamic design optimization on unstructured grids with a continuous adjoint formulation. Computers and Fluids 1999;28(4):443-480.

[23] Jameson A, Martinelli L, Pierce NA. Optimum aerodynamic design using the Navier-Stokes equation. Theorical and Computational Fluid Dynamics 1998;10:213-237.

[24] Anderson WK, Nielsen E. Aerodynamic design optimization on unstructured meshes using the Navier-Stockes equations. AIAA Journal 1999;37(11):1411-1419.

[25] Madsen J. Response surface techniques for diffuser shape optimization. AIAA Journal 2000; 38(9):1512-1518.

[26] Ravindran S. Active control of flow separation over an airfoil. Technical Report TM-1999-209838, NASA 1999. 


\section{List of Figures}

Grid assessment . . . . . . . . . . . . . . . . . . . . . 14

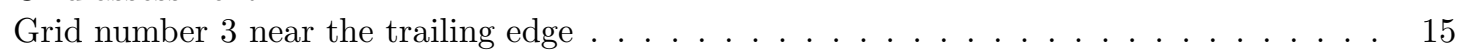

Lift coef. with respect to the incidence . . . . . . . . . . . . . . . . 16

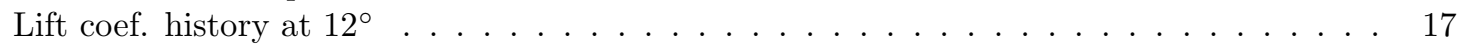

Lift coef. history at $14^{\circ} \ldots \ldots \ldots \ldots \ldots \ldots \ldots \ldots \ldots$

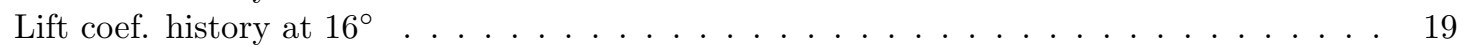

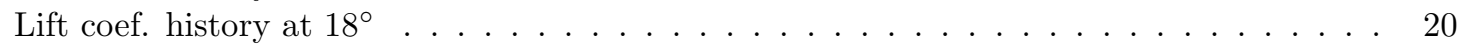

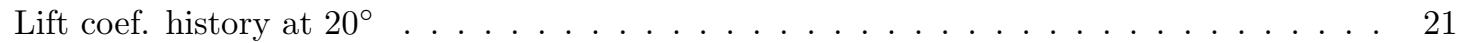

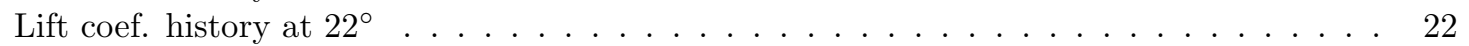

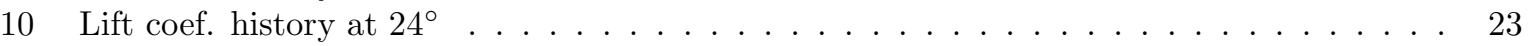

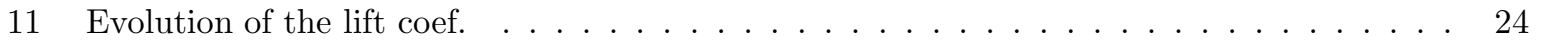

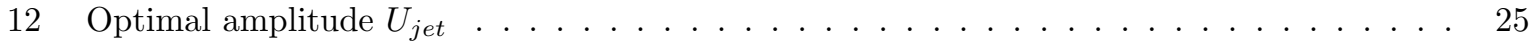

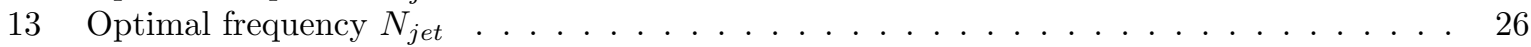

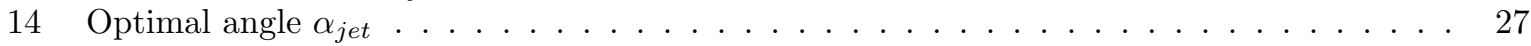

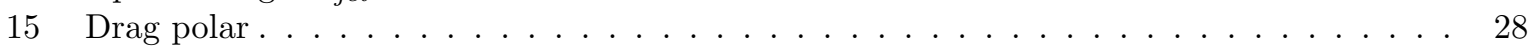

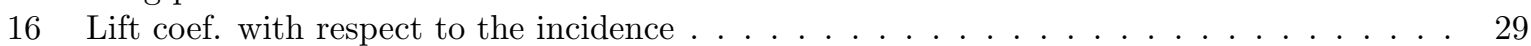

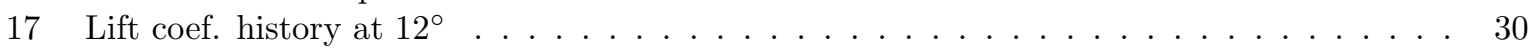

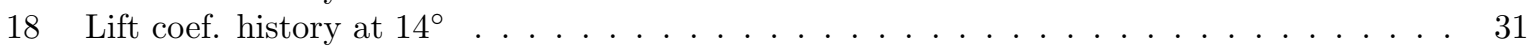

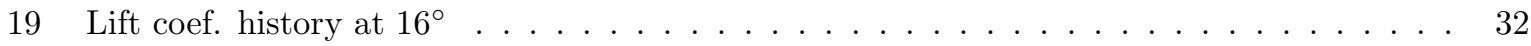

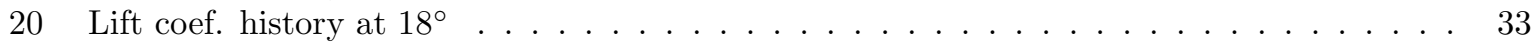

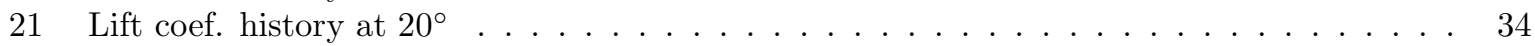

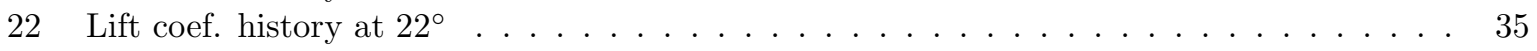

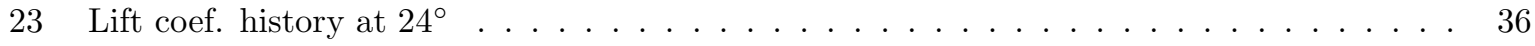

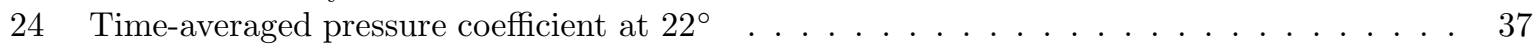

25 Time-averaged pressure coefficient at $22^{\circ}$ (zoom) $\ldots \ldots \ldots \ldots \ldots$

26 Streamlines for baseline airfoil . . . . . . . . . . . . . . . . . . . . . . 39

27 Streamlines with initial parameters . . . . . . . . . . . . . . . . . . 40

28 Streamlines with mean optimal parameters . . . . . . . . . . . . . . . . . 41

29 Streamlines with optimal parameters at $22^{\circ} \ldots \ldots \ldots \ldots \ldots$. . . . . . . . . 42 


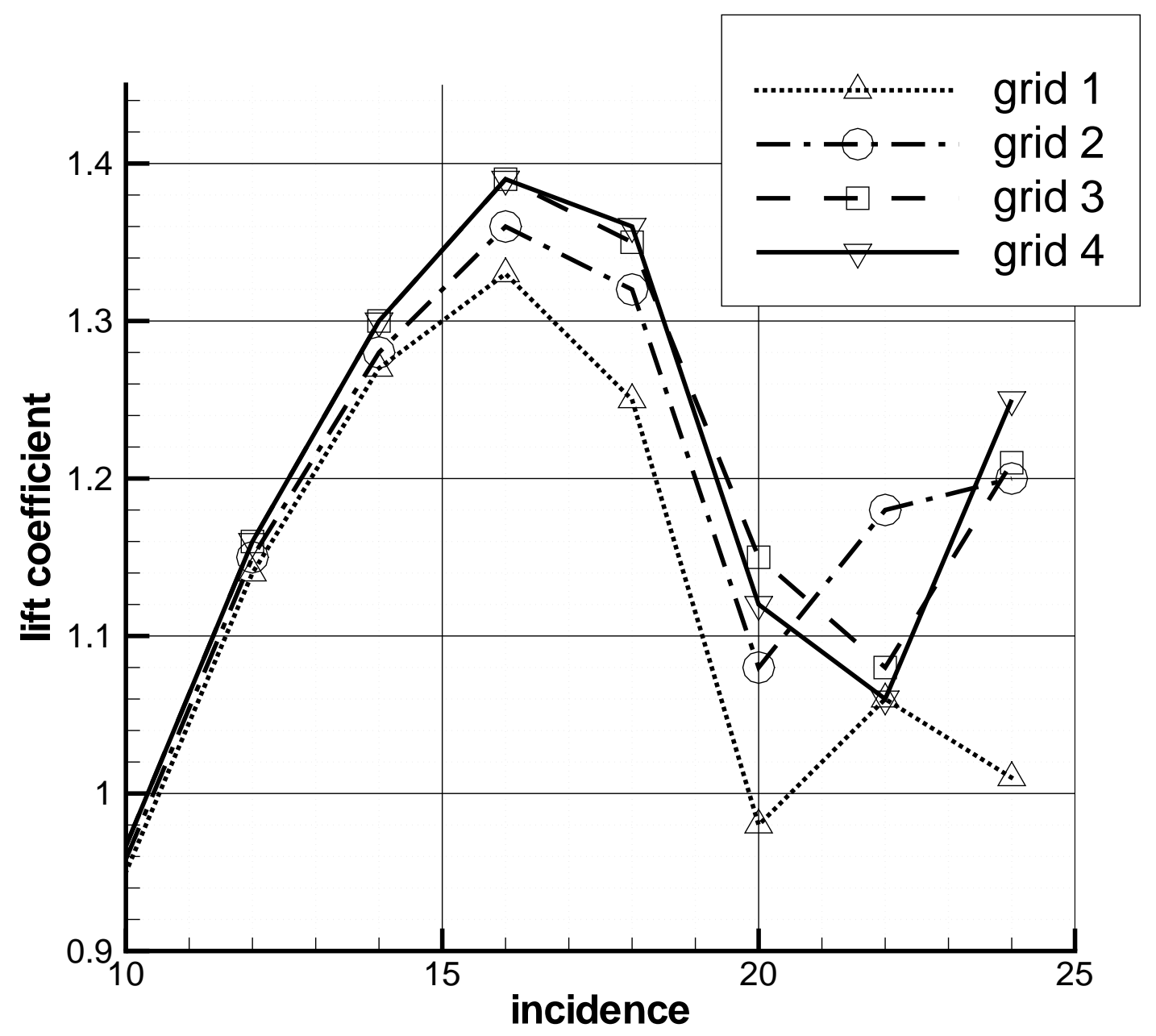

Figure 1: Grid assessment 


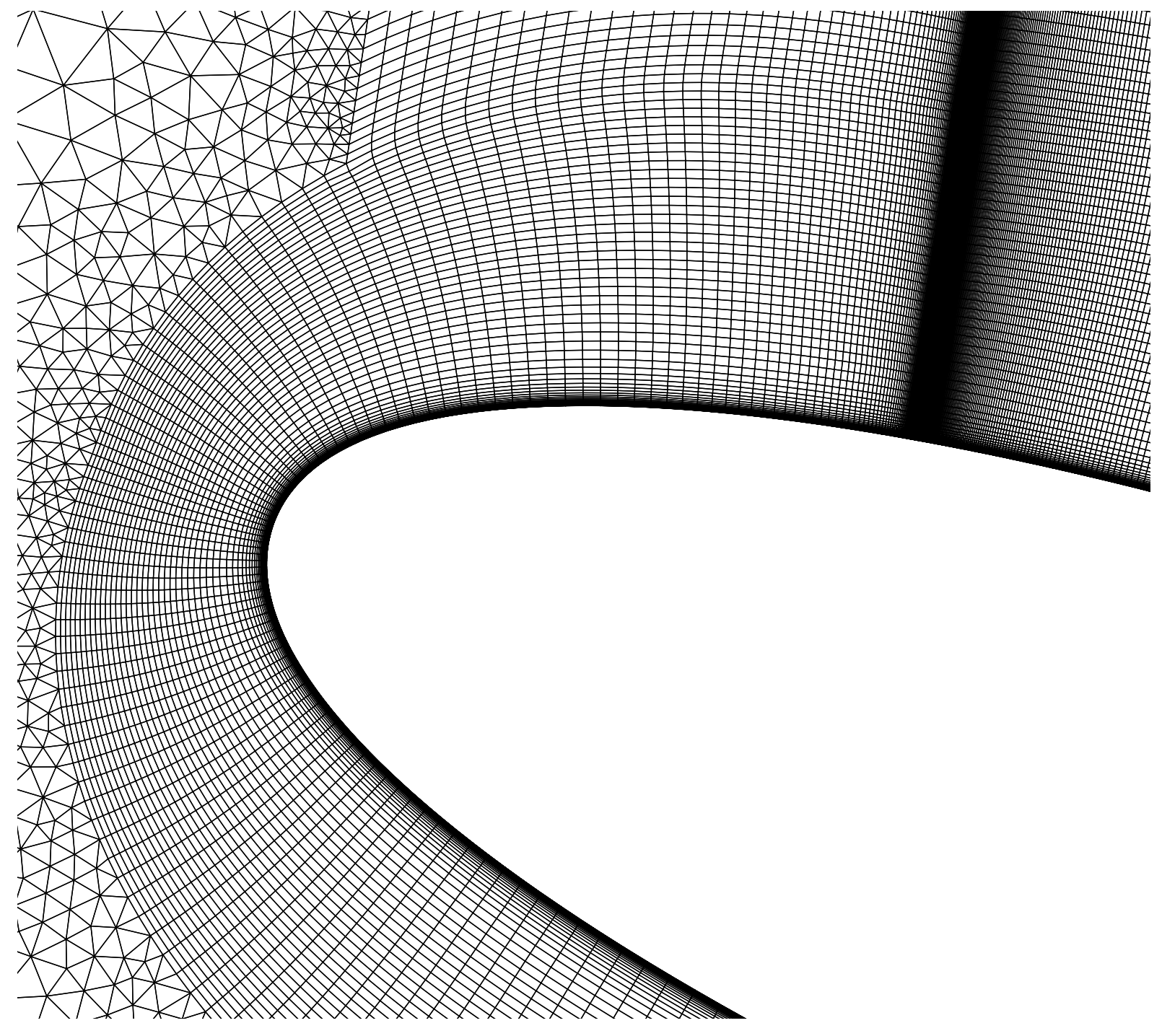

Figure 2: Grid number 3 near the trailing edge 


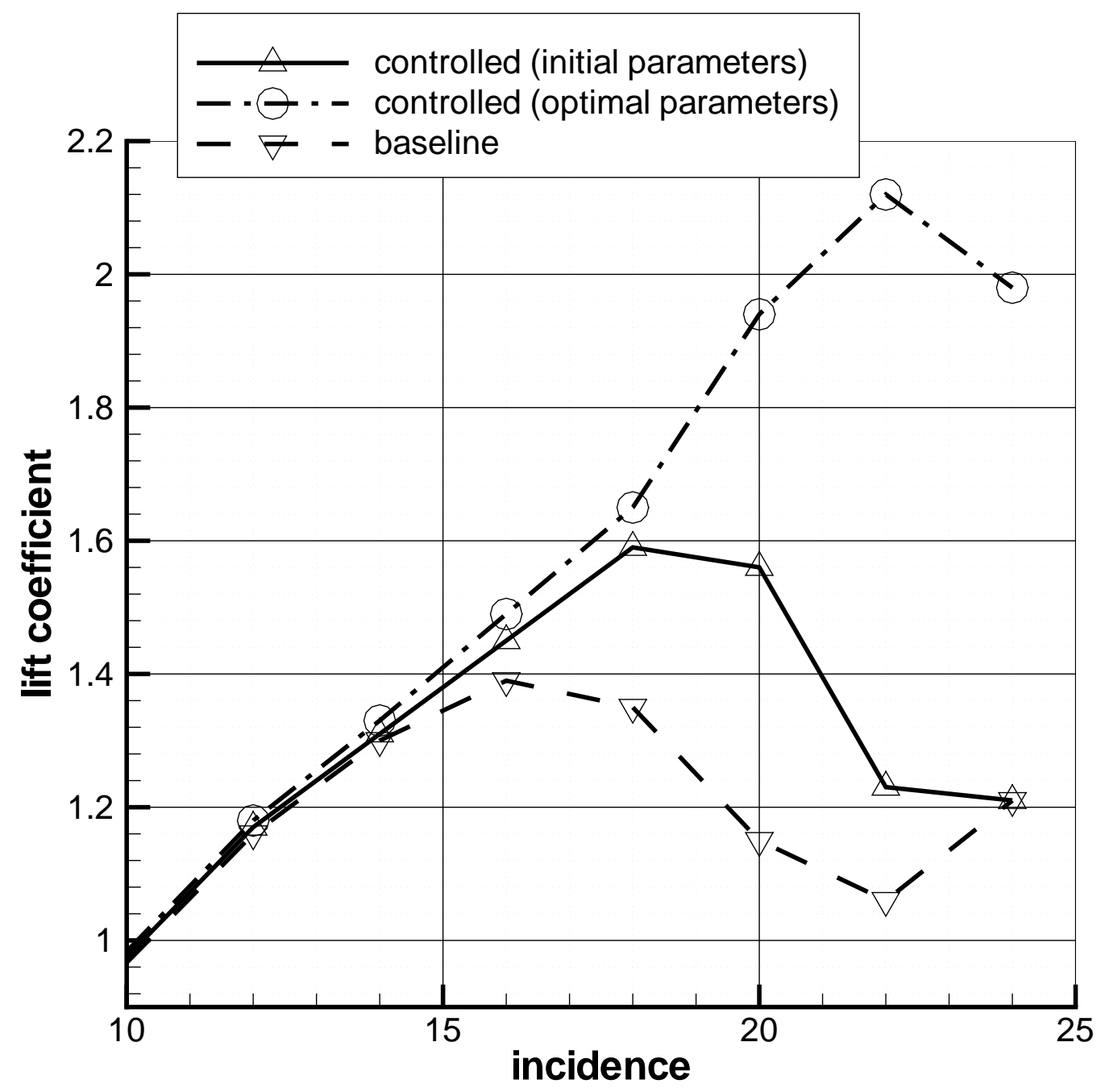

Figure 3: Lift coef. with respect to the incidence 


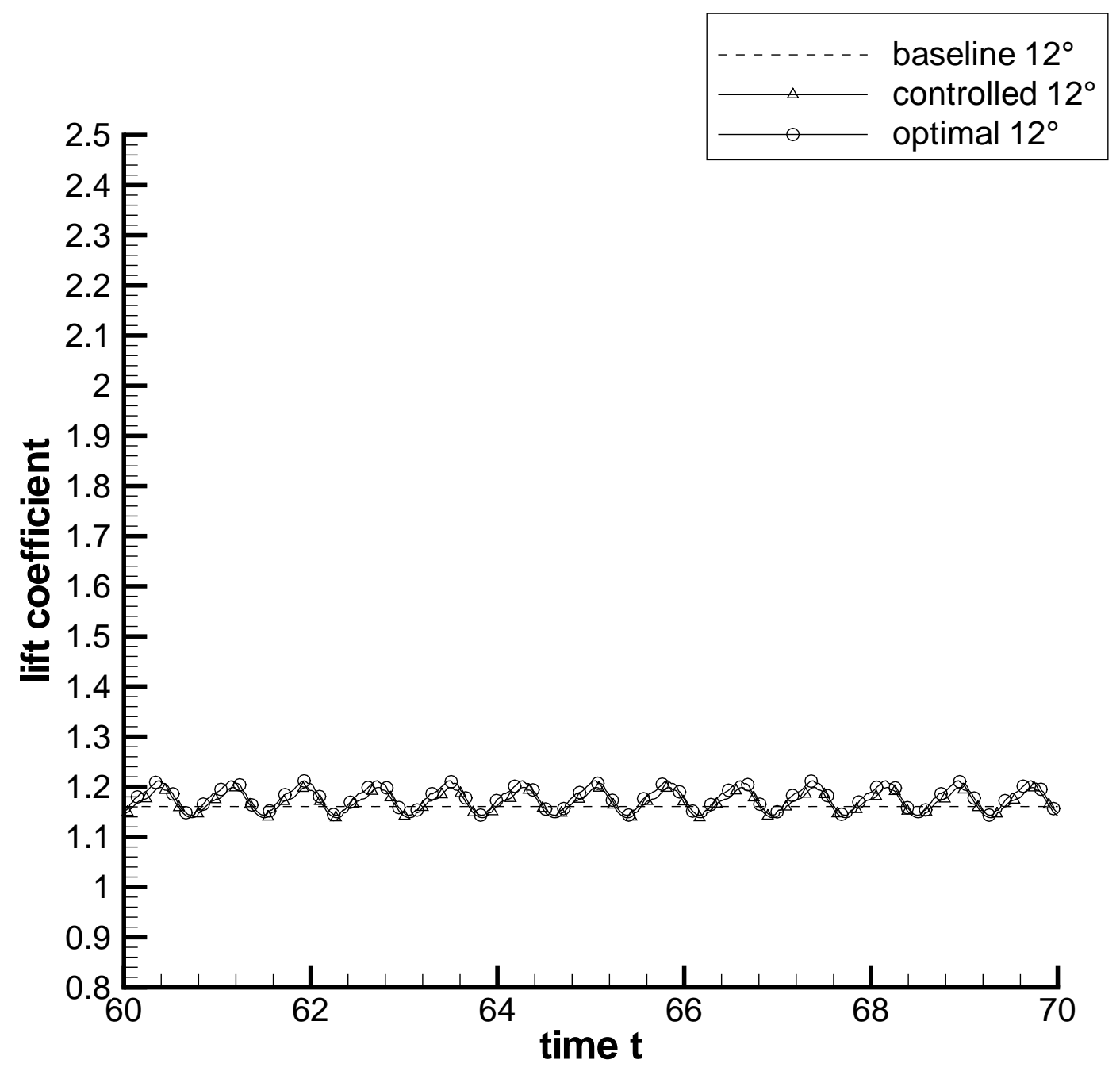

Figure 4: Lift coef. history at $12^{\circ}$ 


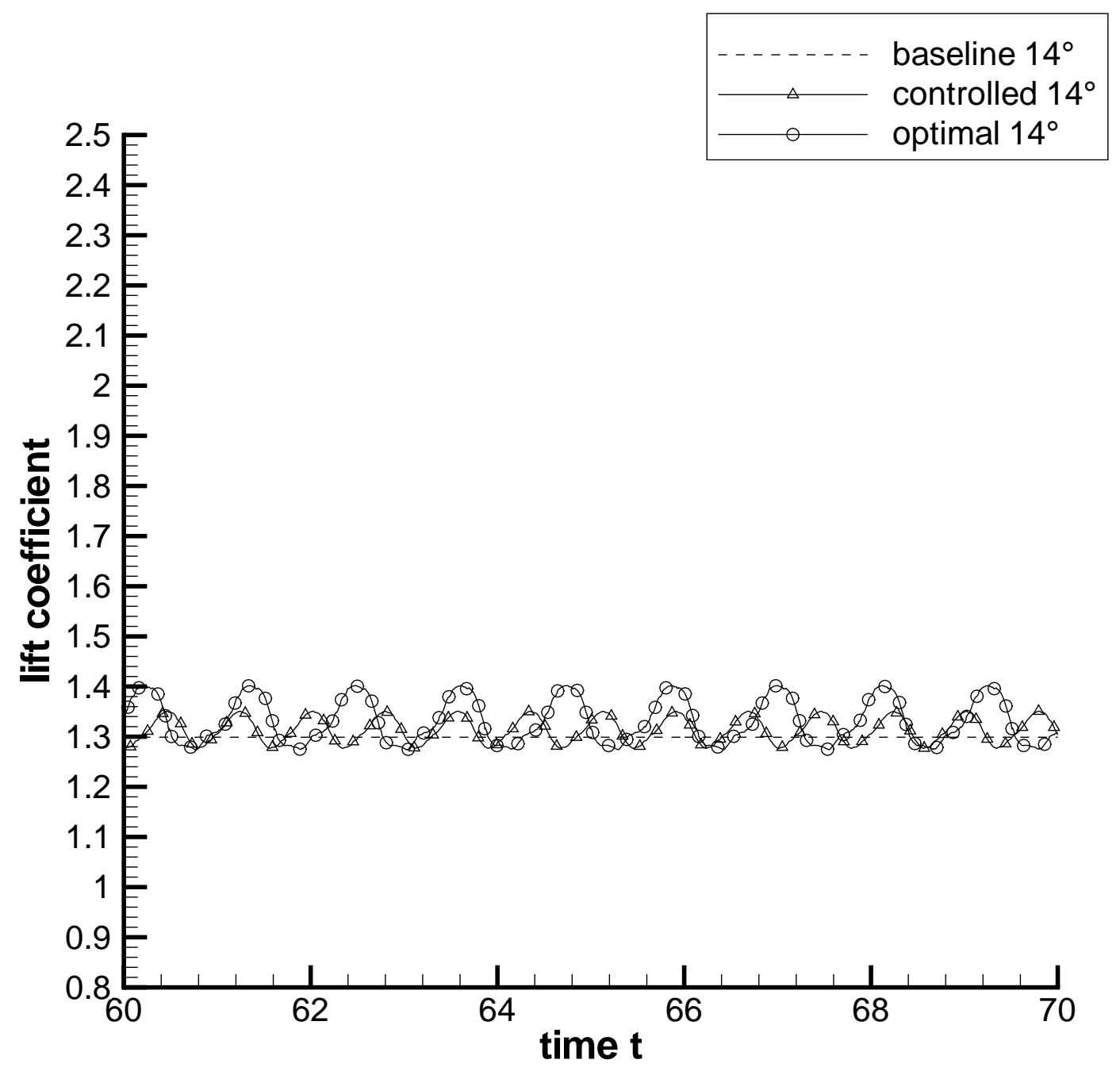

Figure 5: Lift coef. history at $14^{\circ}$ 


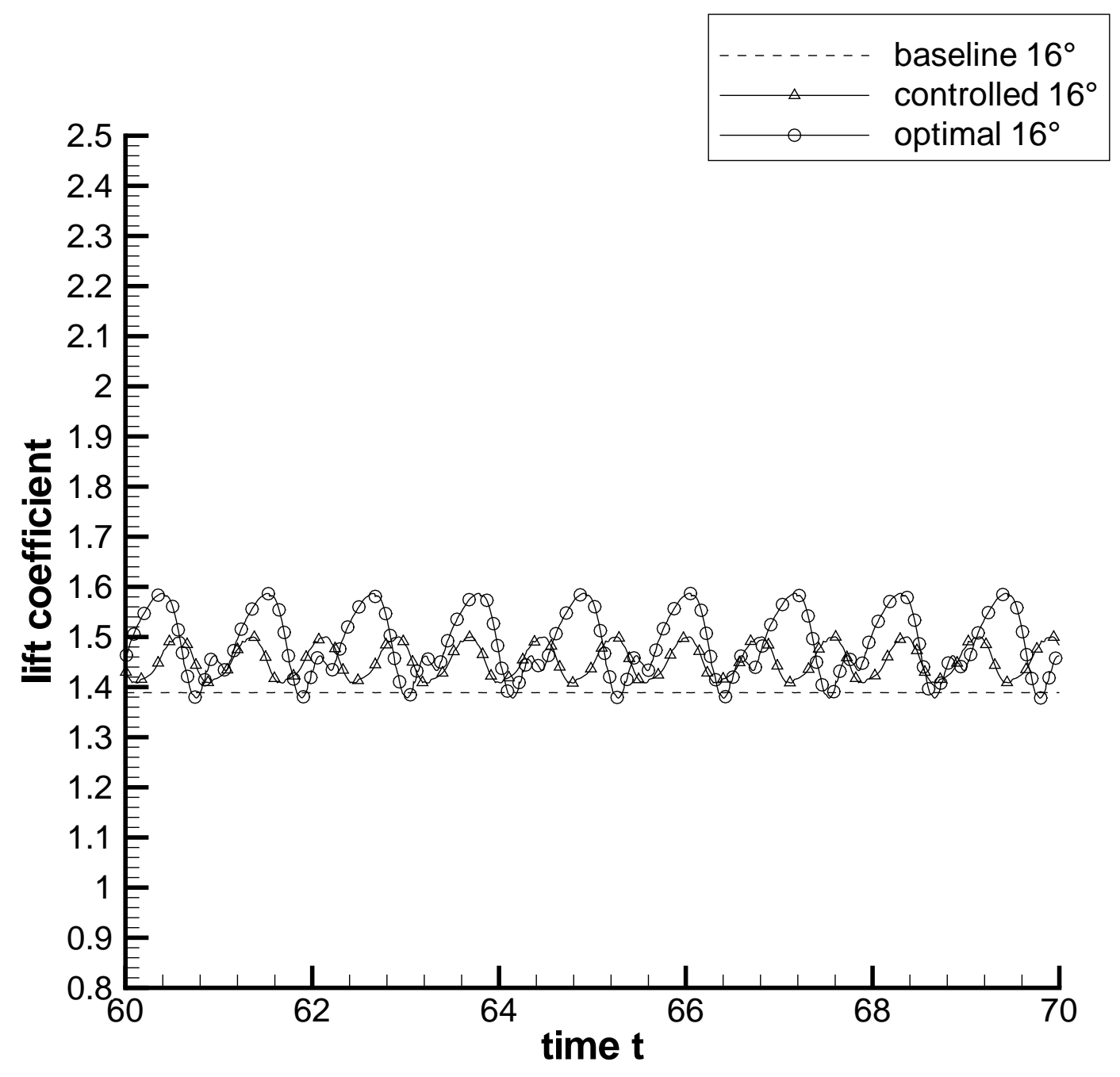

Figure 6: Lift coef. history at $16^{\circ}$ 


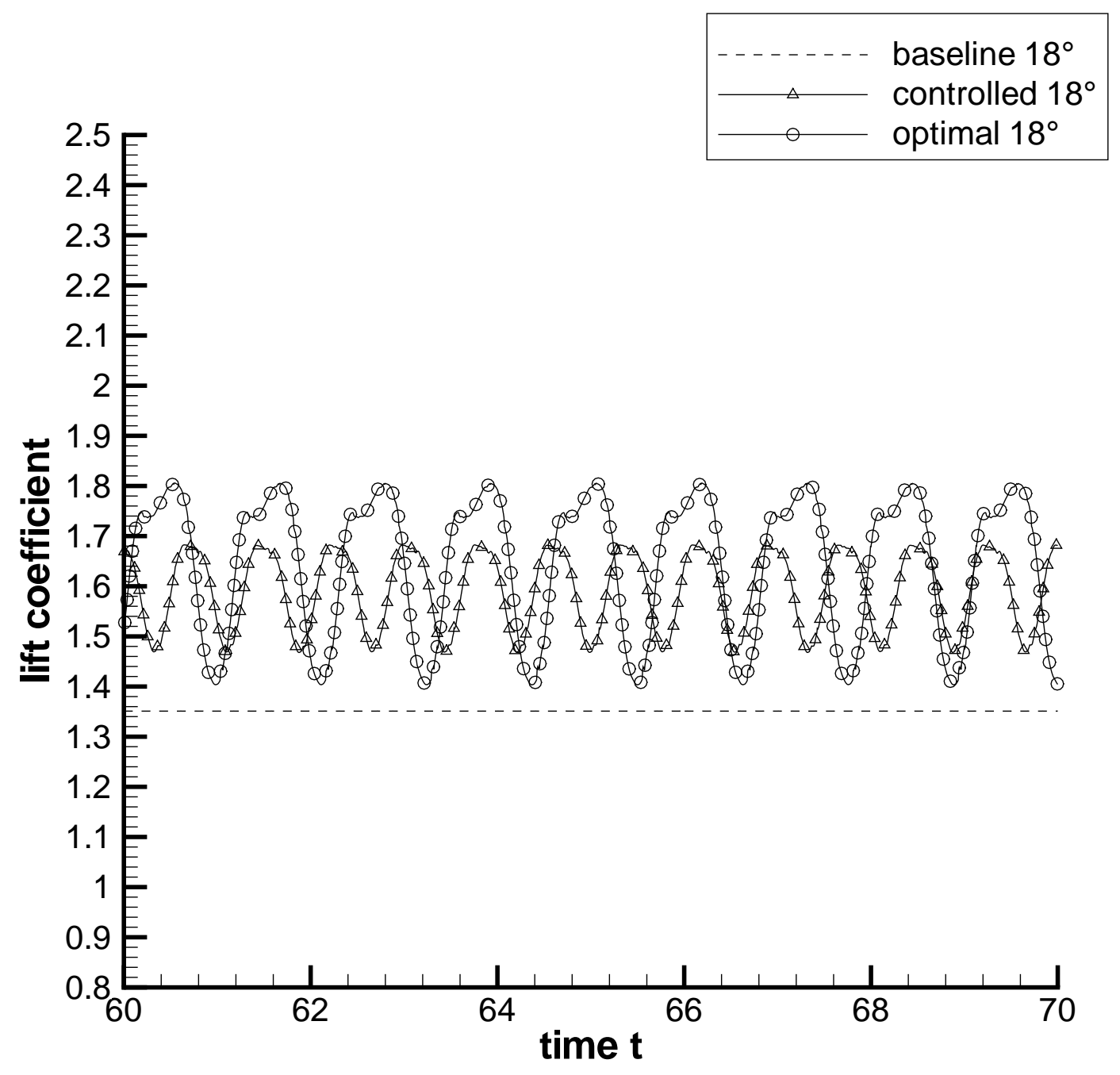

Figure 7: Lift coef. history at $18^{\circ}$ 


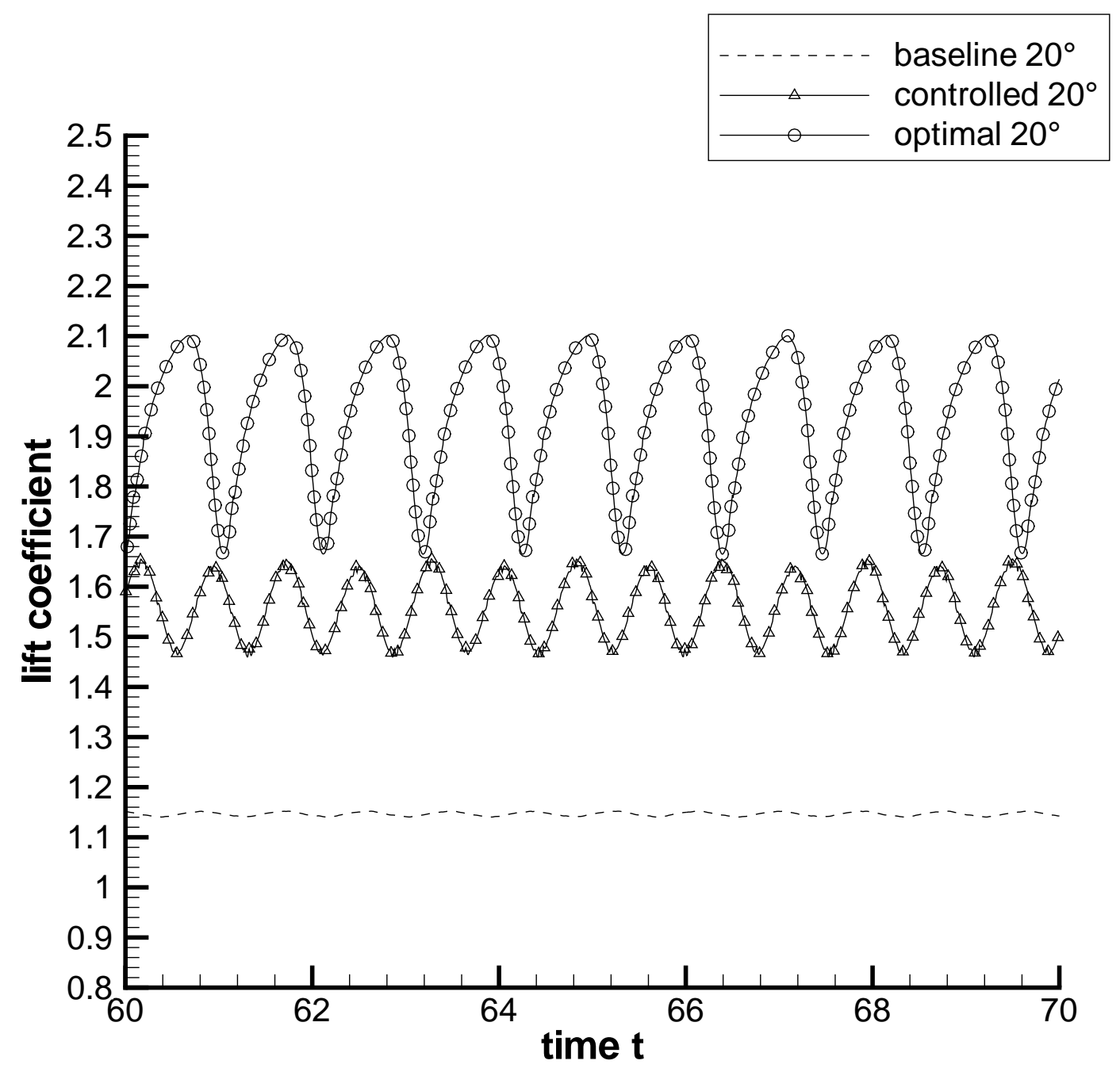

Figure 8: Lift coef. history at $20^{\circ}$ 


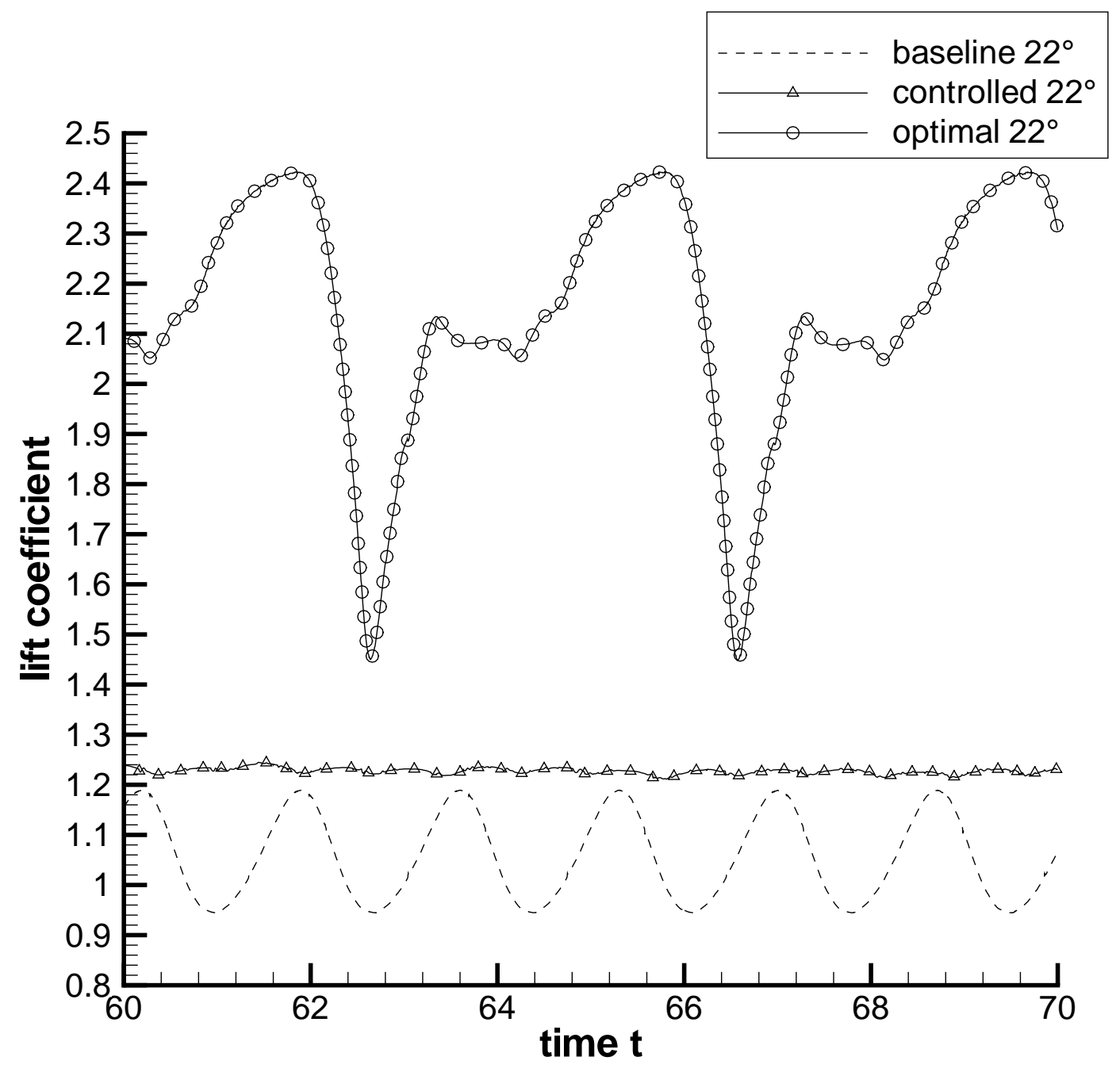

Figure 9: Lift coef. history at $22^{\circ}$ 


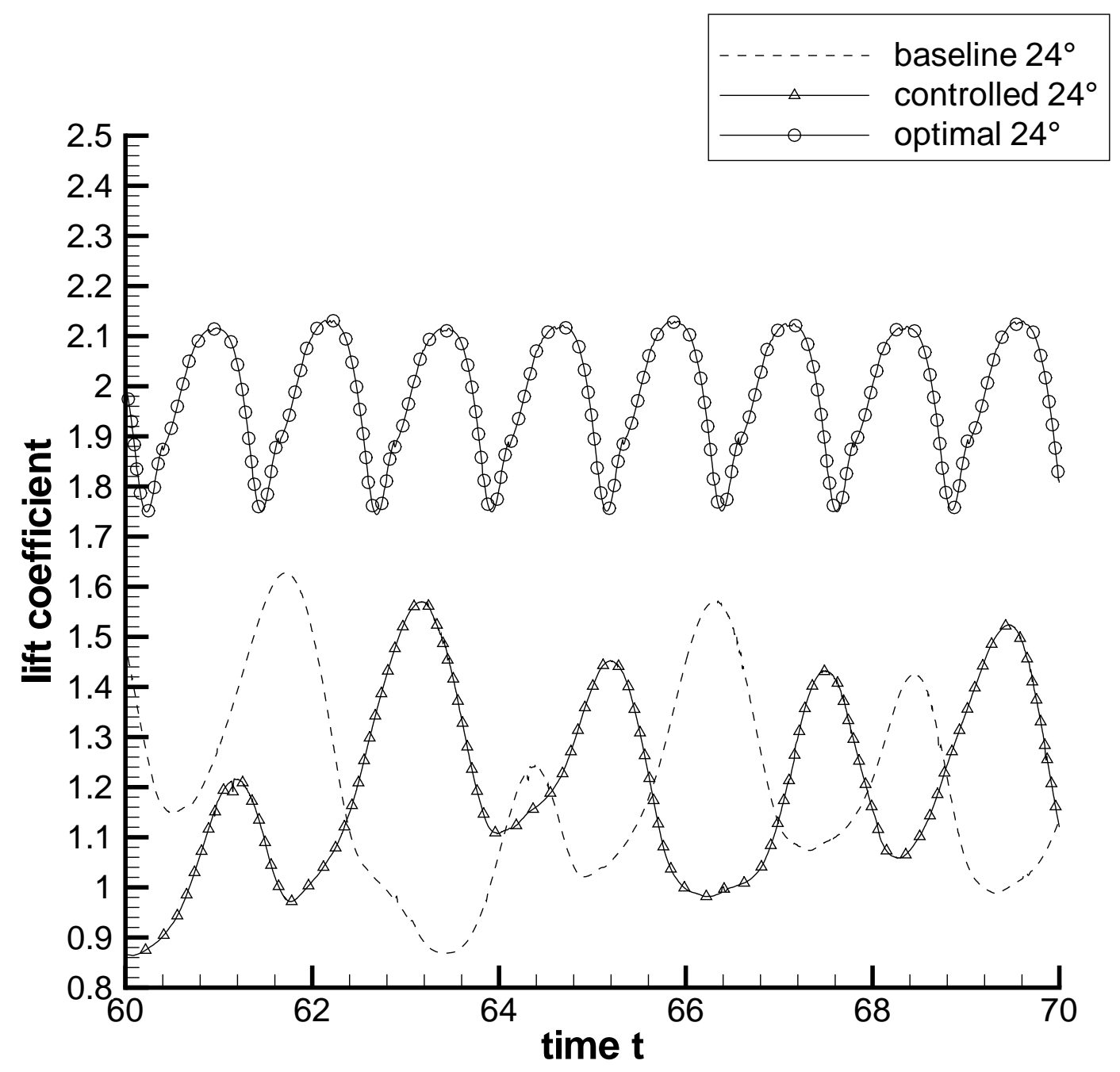

Figure 10: Lift coef. history at $24^{\circ}$ 


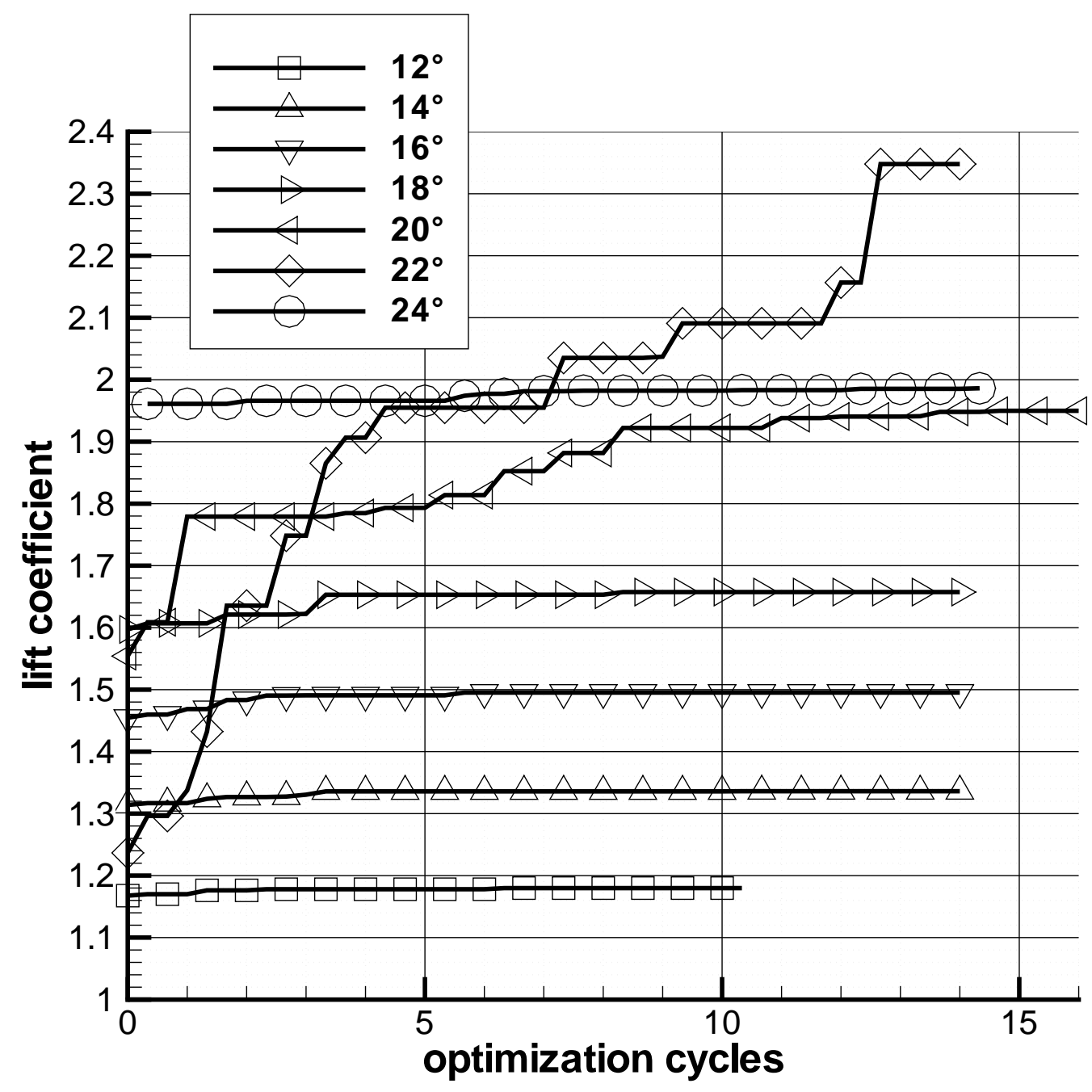

Figure 11: Evolution of the lift coef. 


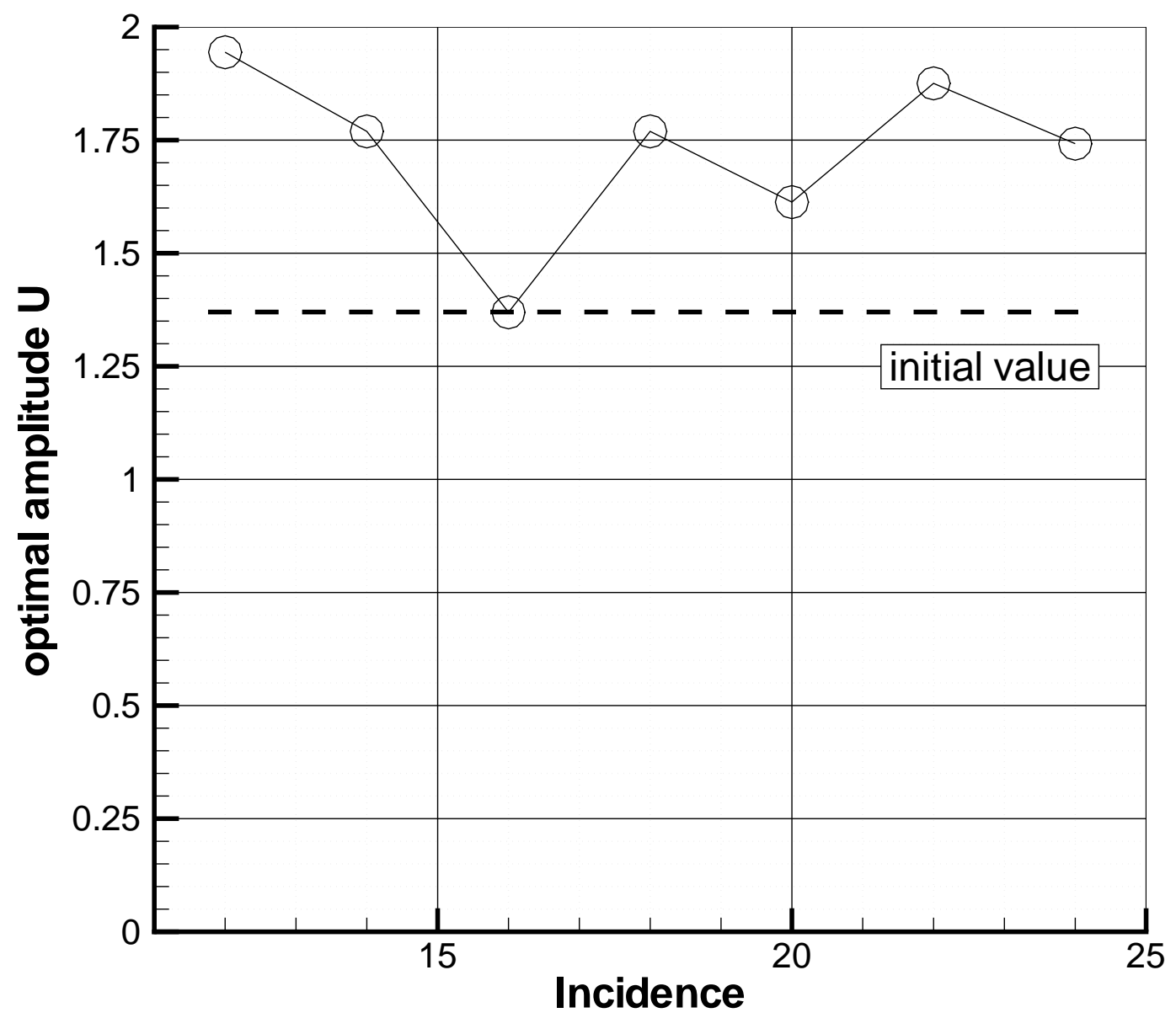

Figure 12: Optimal amplitude $U_{\text {jet }}$ 


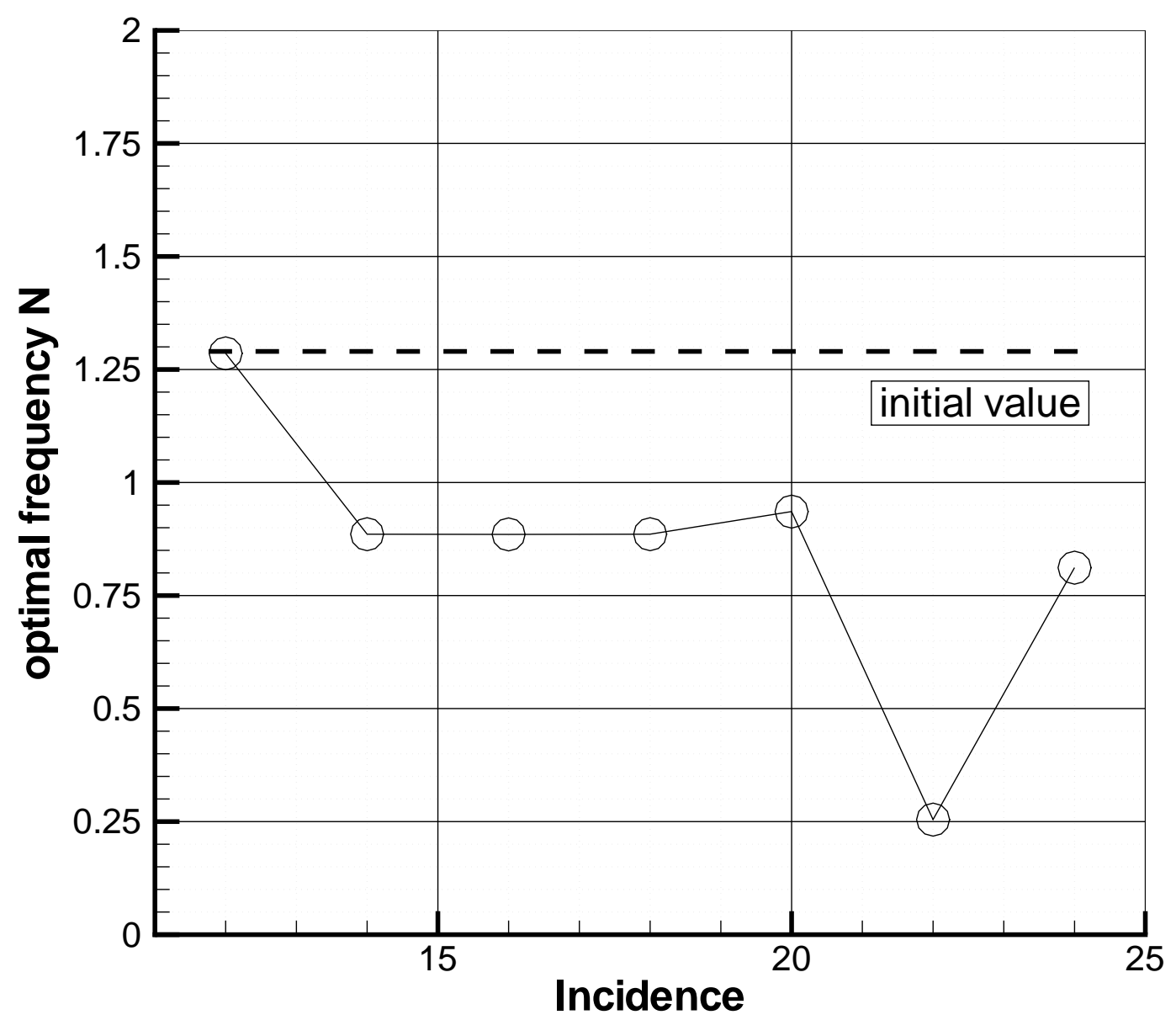

Figure 13: Optimal frequency $N_{\text {jet }}$ 


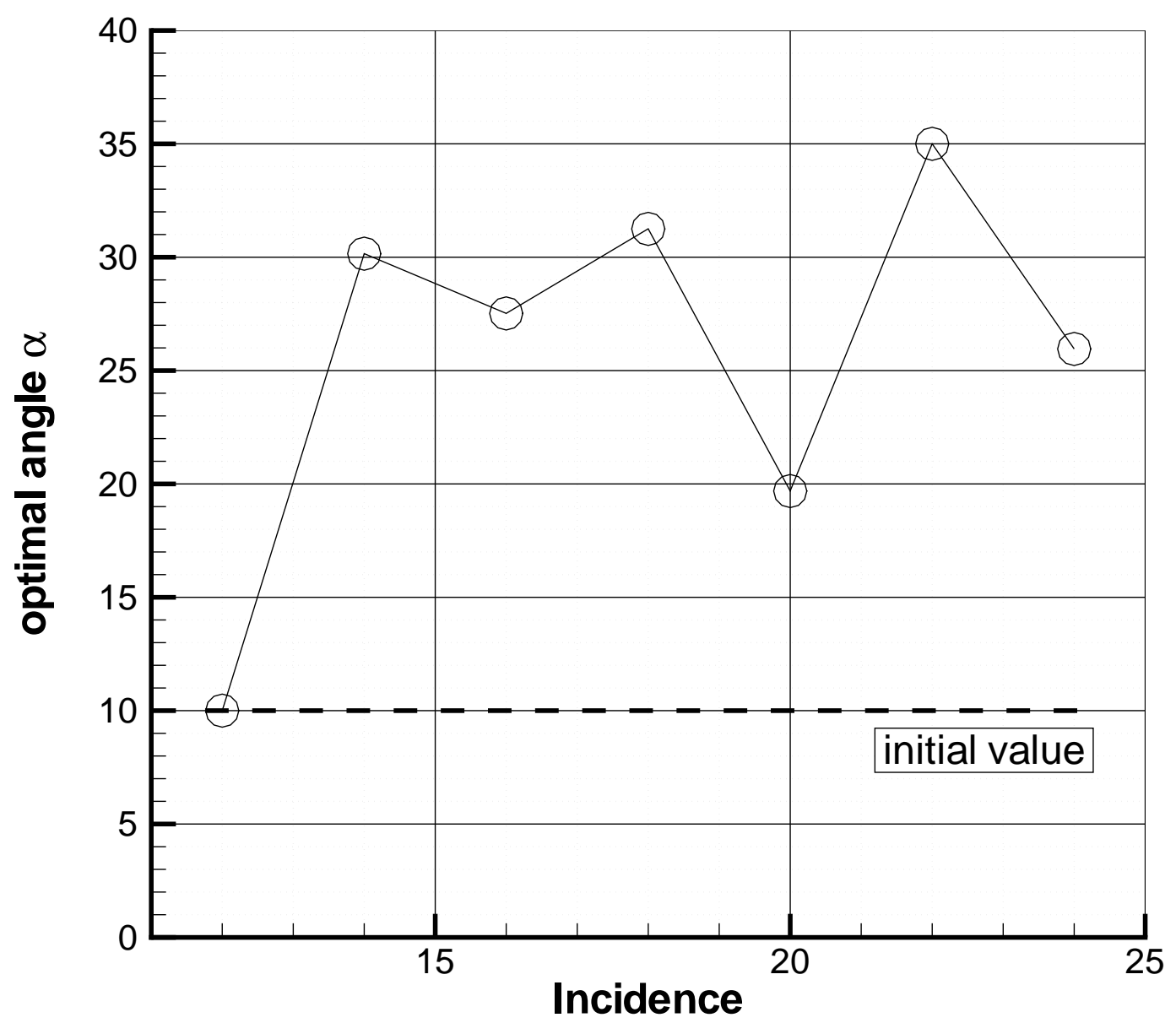

Figure 14: Optimal angle $\alpha_{j e t}$ 


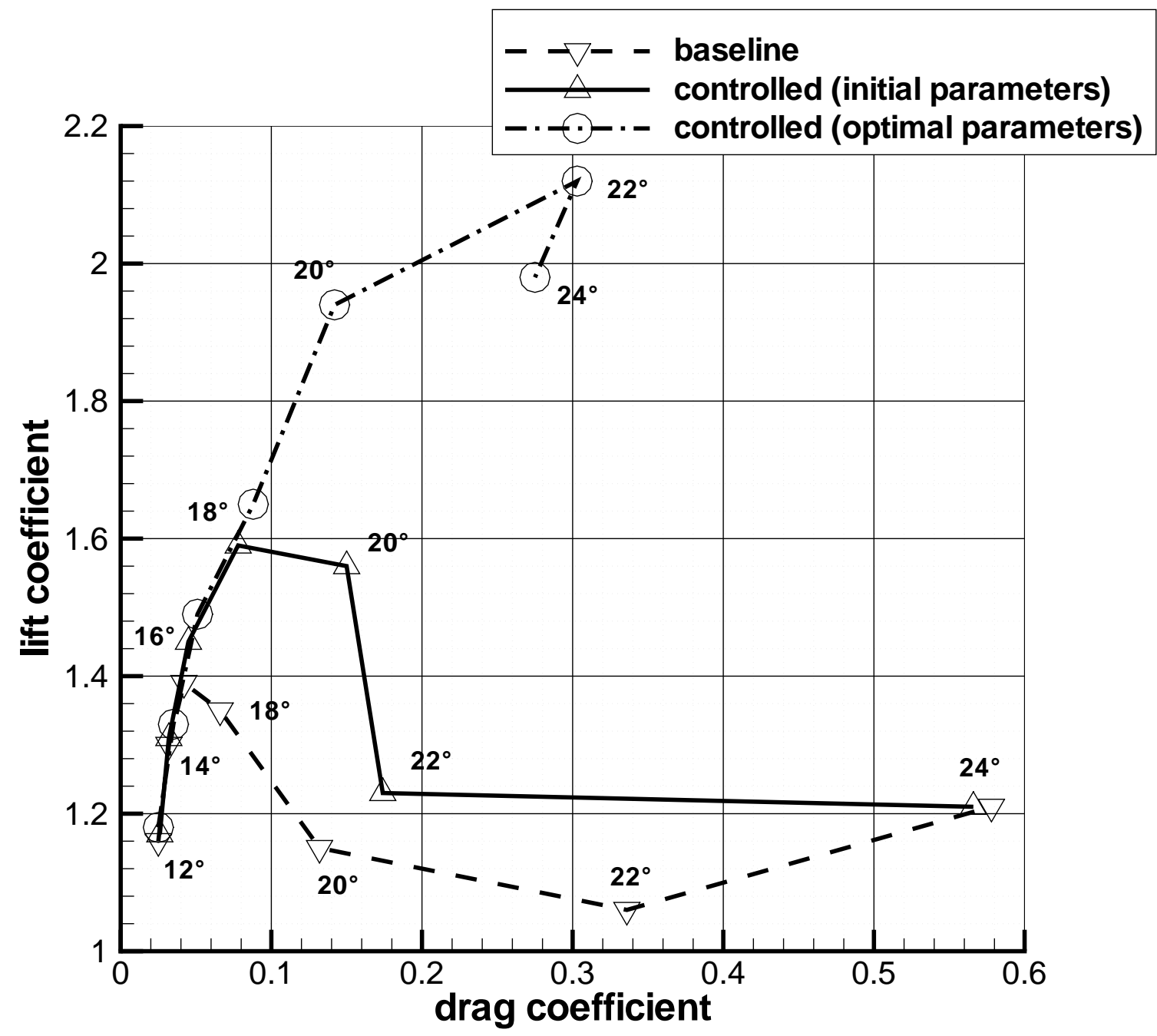

Figure 15: Drag polar 


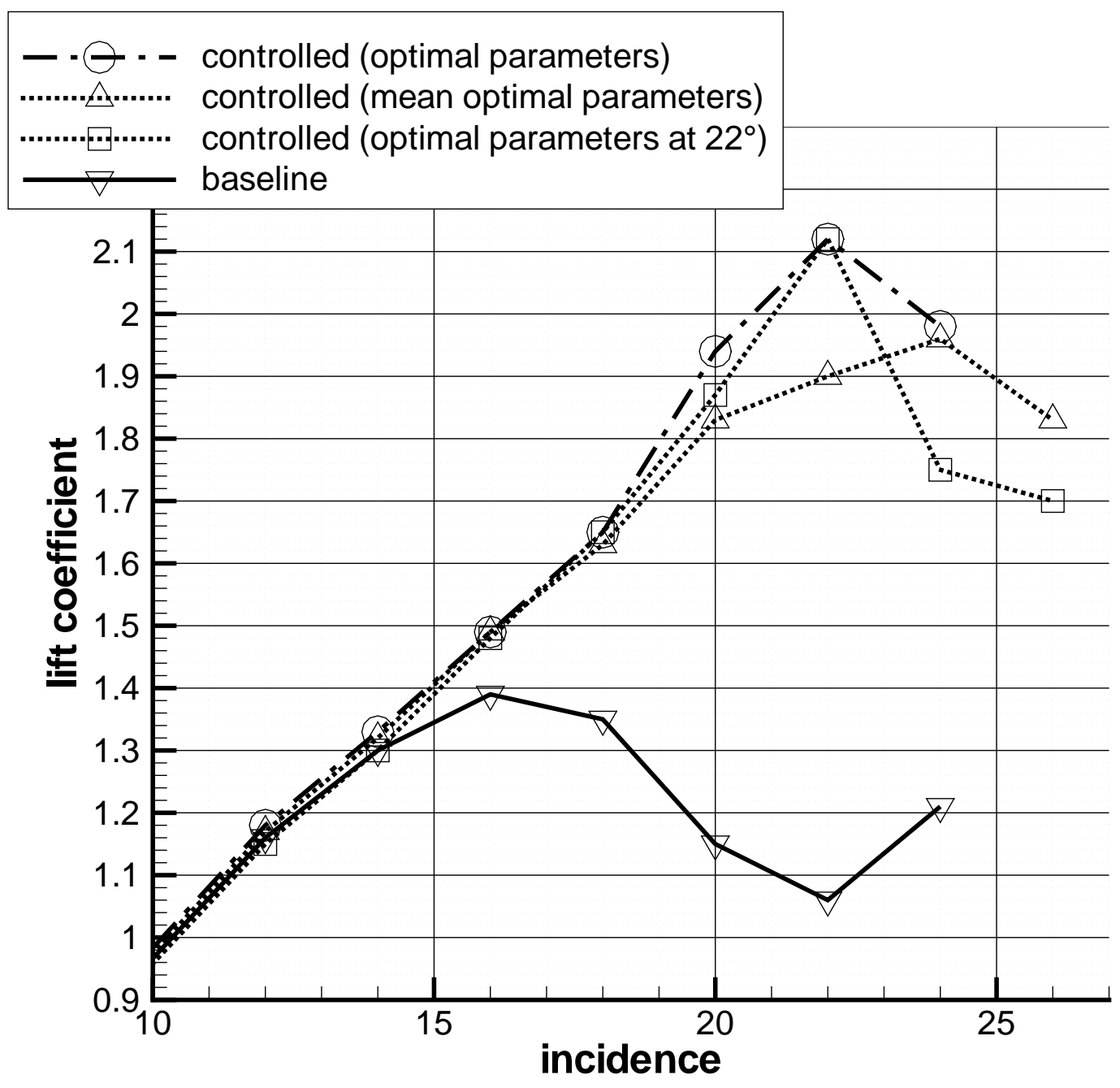

Figure 16: Lift coef. with respect to the incidence 


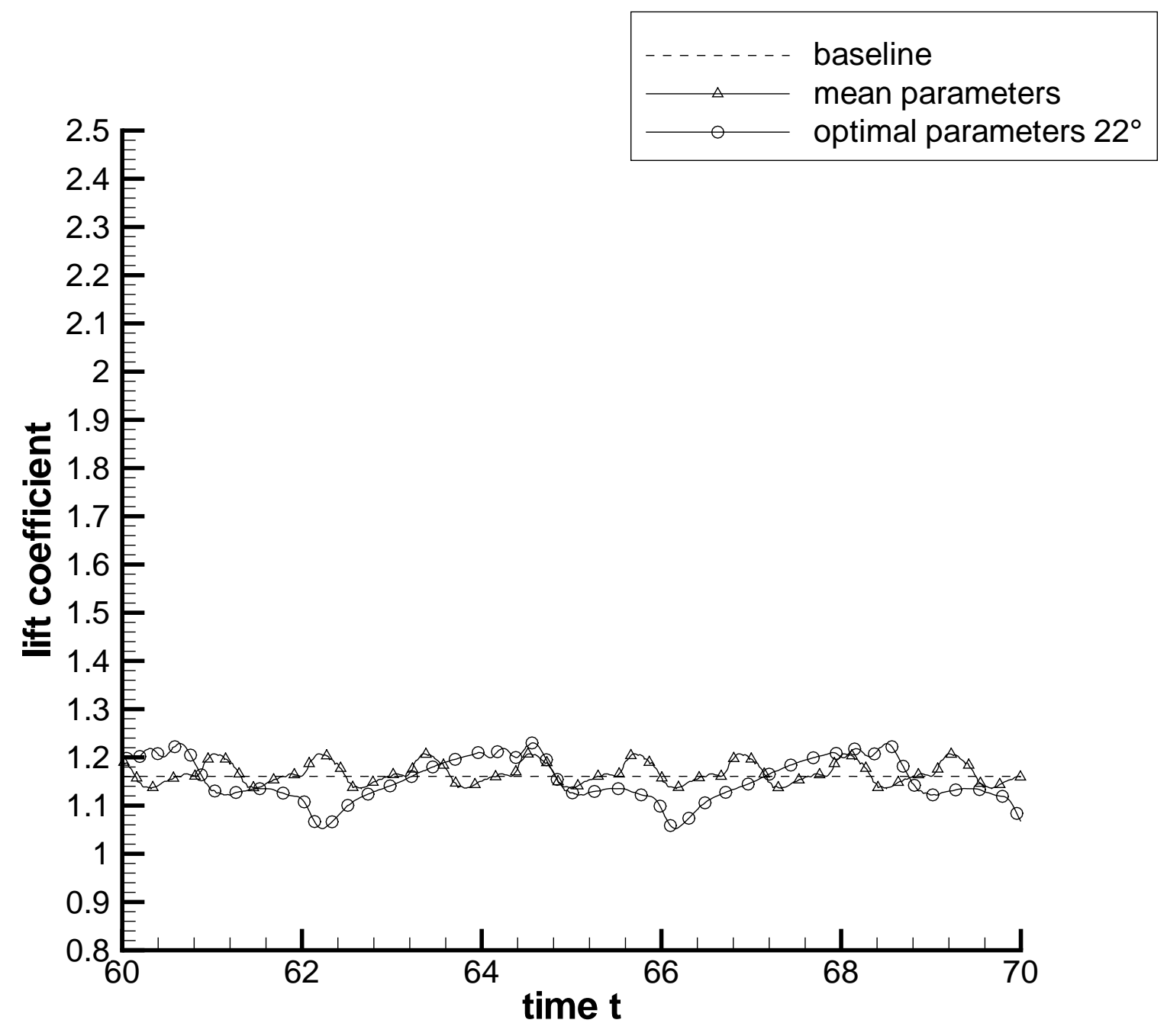

Figure 17: Lift coef. history at $12^{\circ}$ 


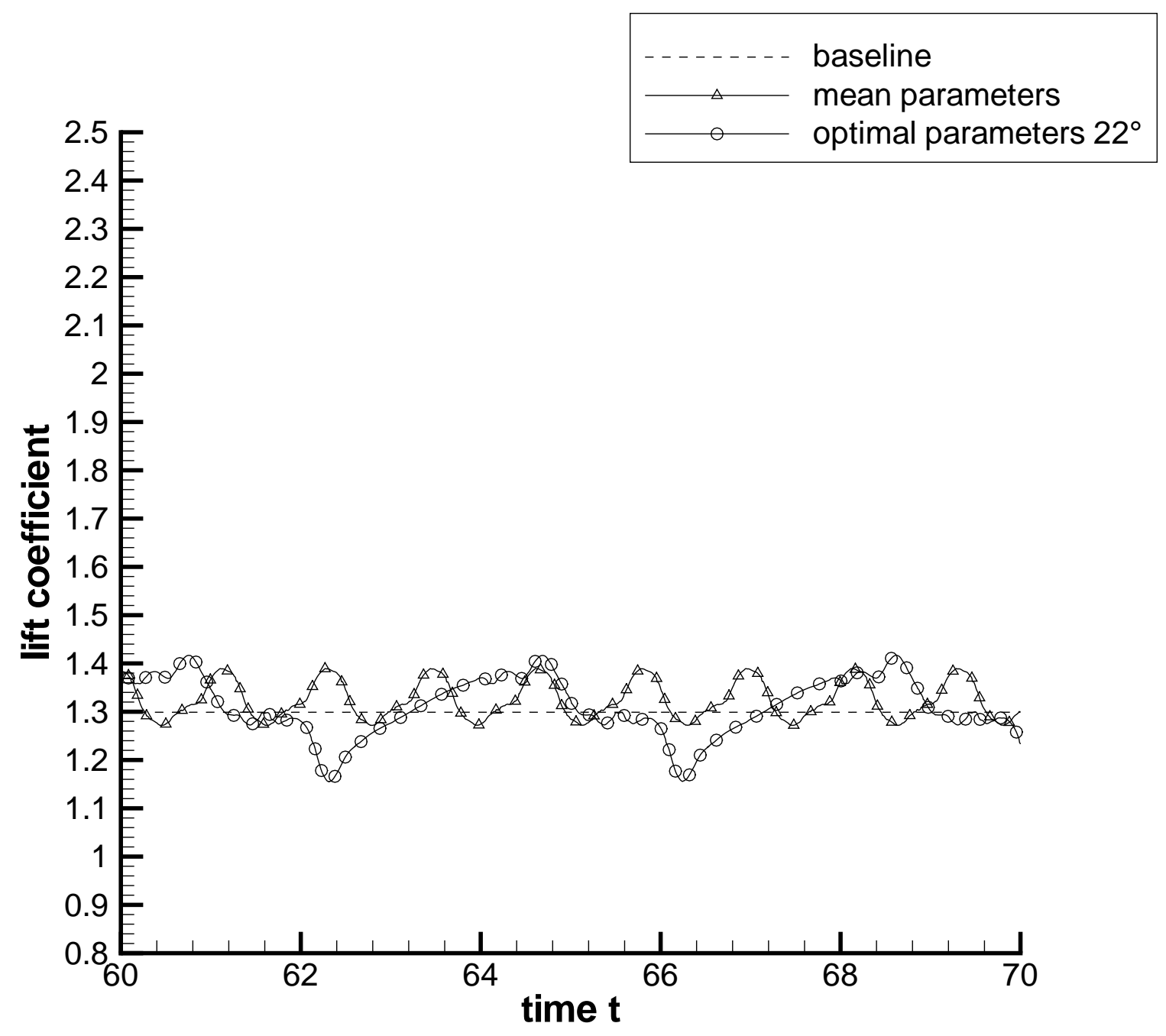

Figure 18: Lift coef. history at $14^{\circ}$ 


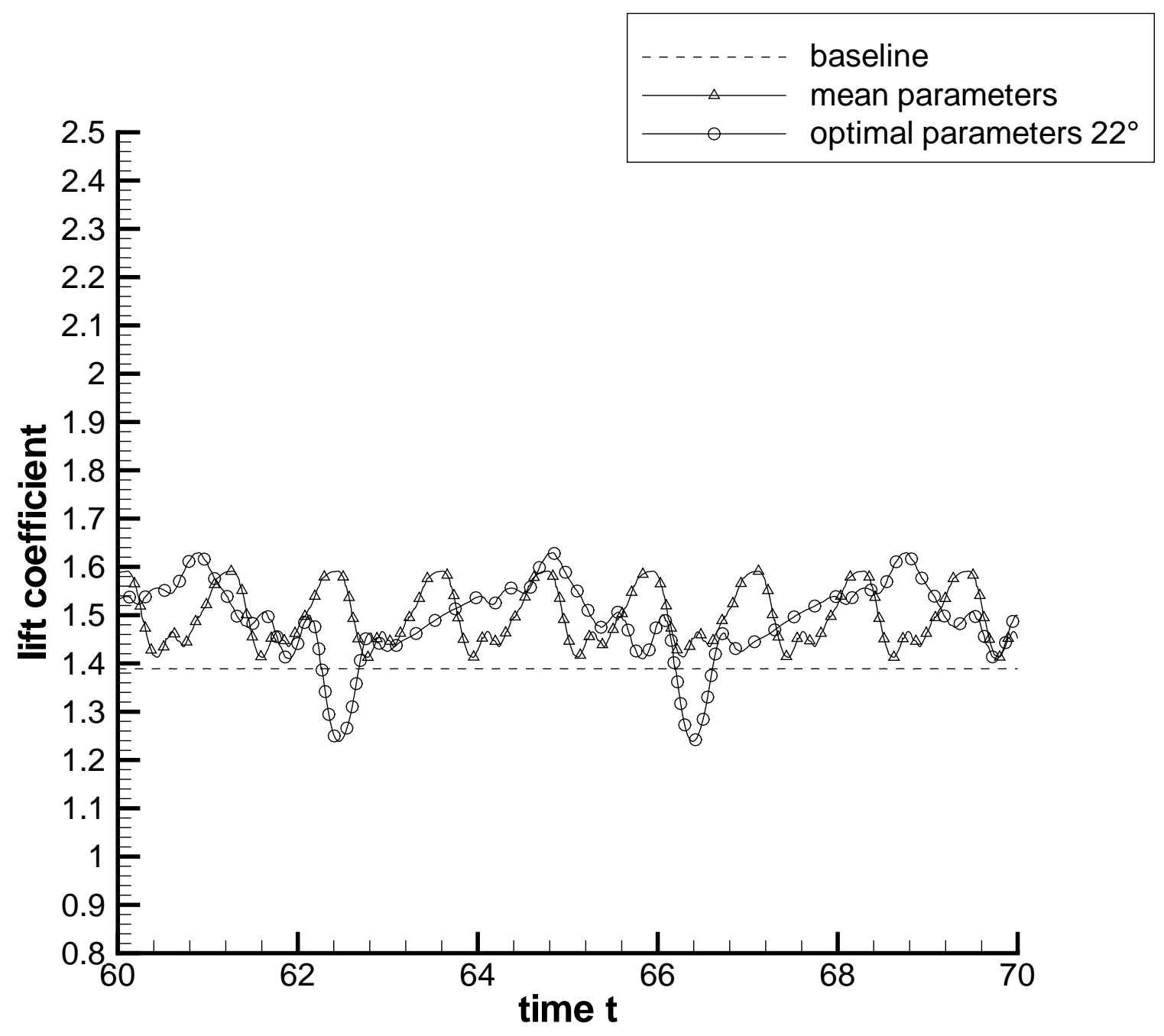

Figure 19: Lift coef. history at $16^{\circ}$ 


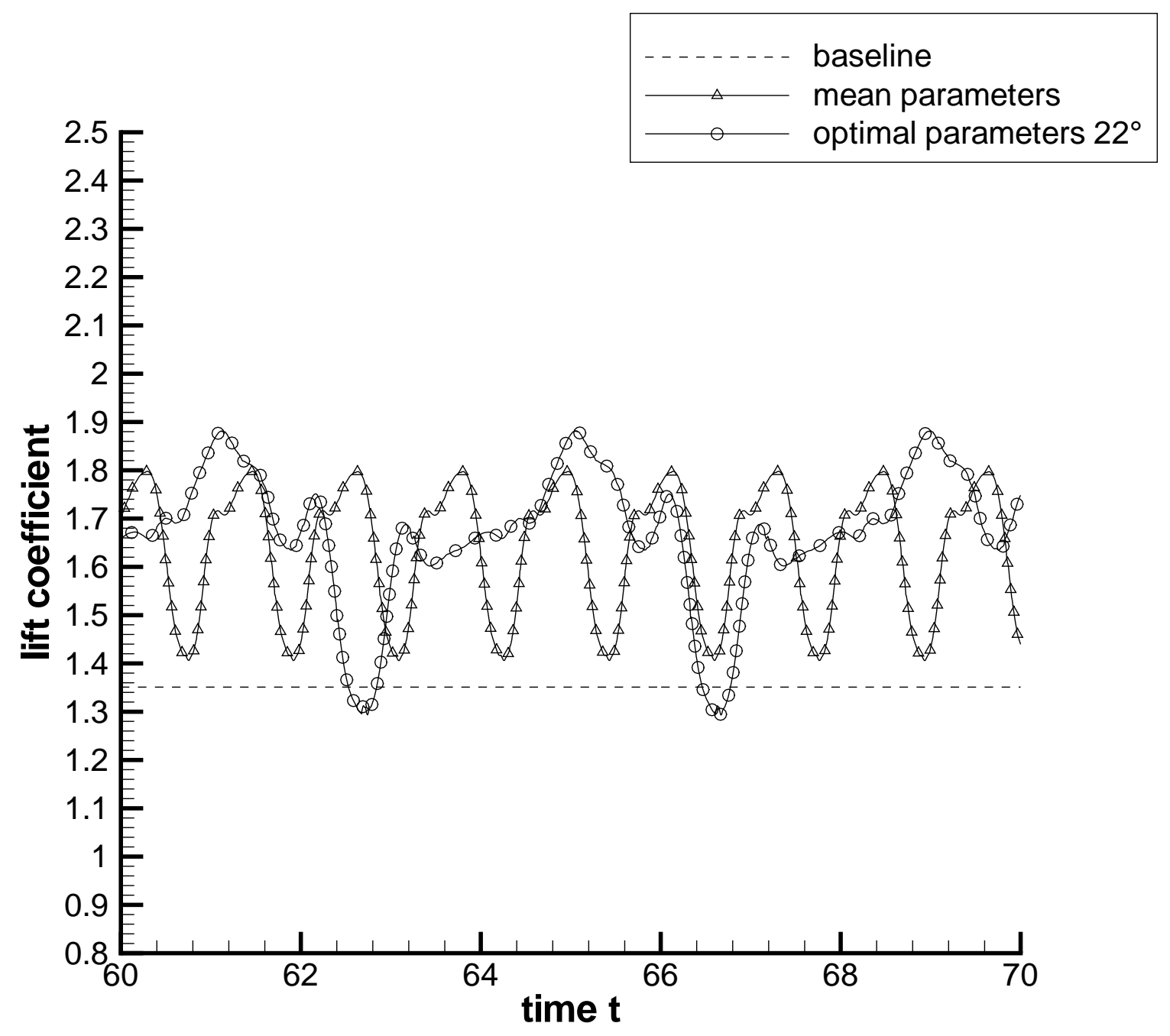

Figure 20: Lift coef. history at $18^{\circ}$ 


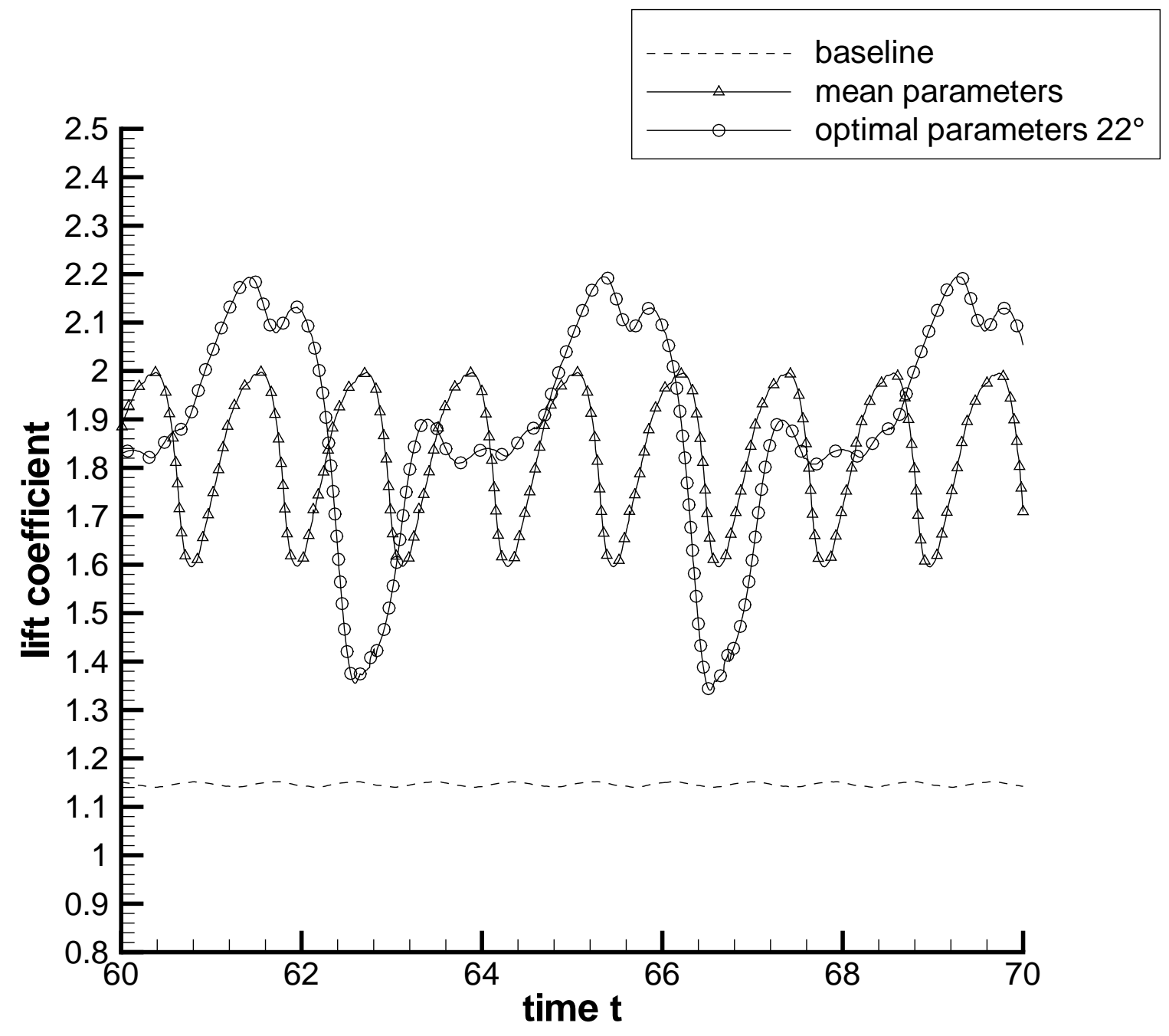

Figure 21: Lift coef. history at $20^{\circ}$ 


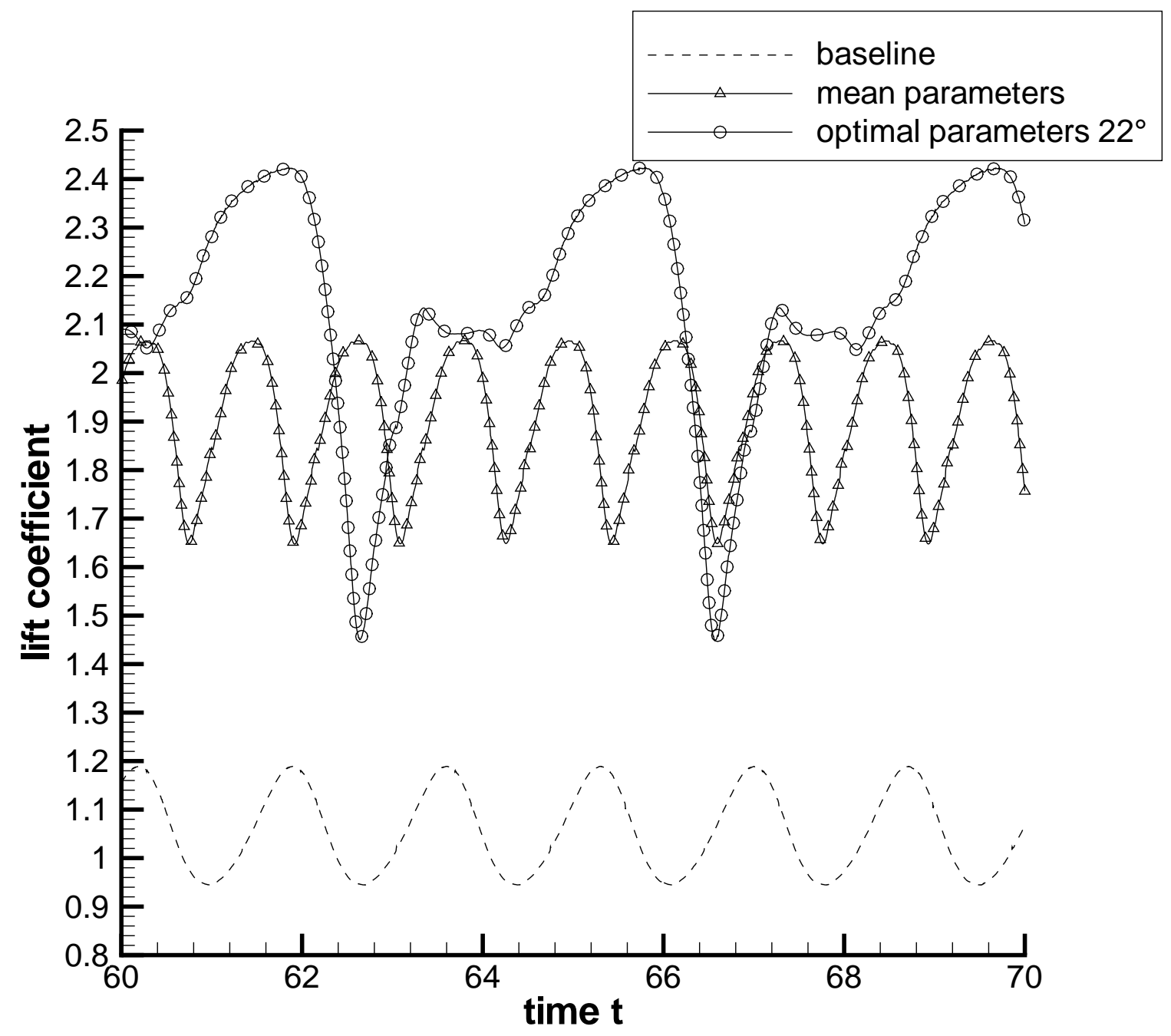

Figure 22: Lift coef. history at $22^{\circ}$ 


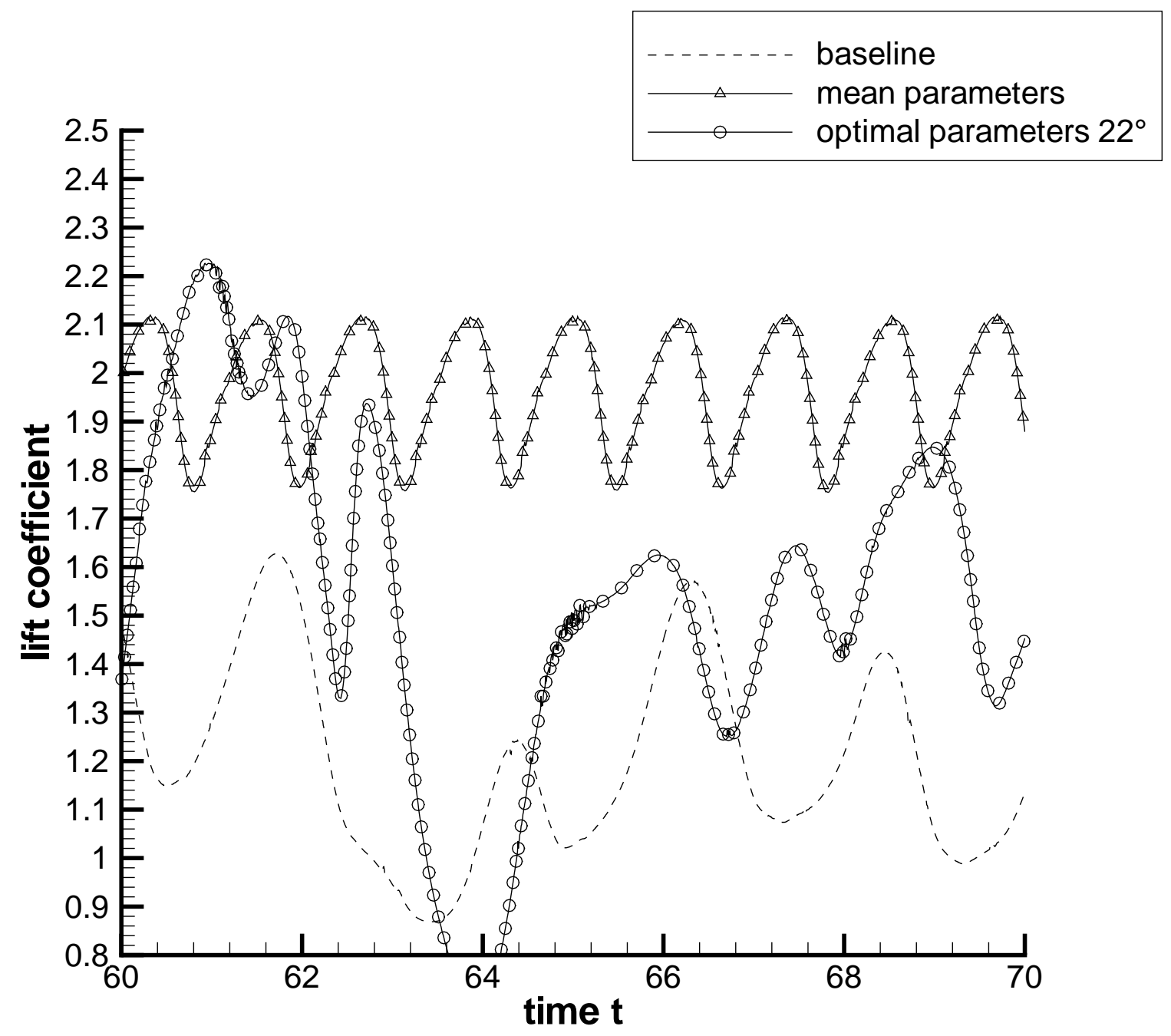

Figure 23: Lift coef. history at $24^{\circ}$ 


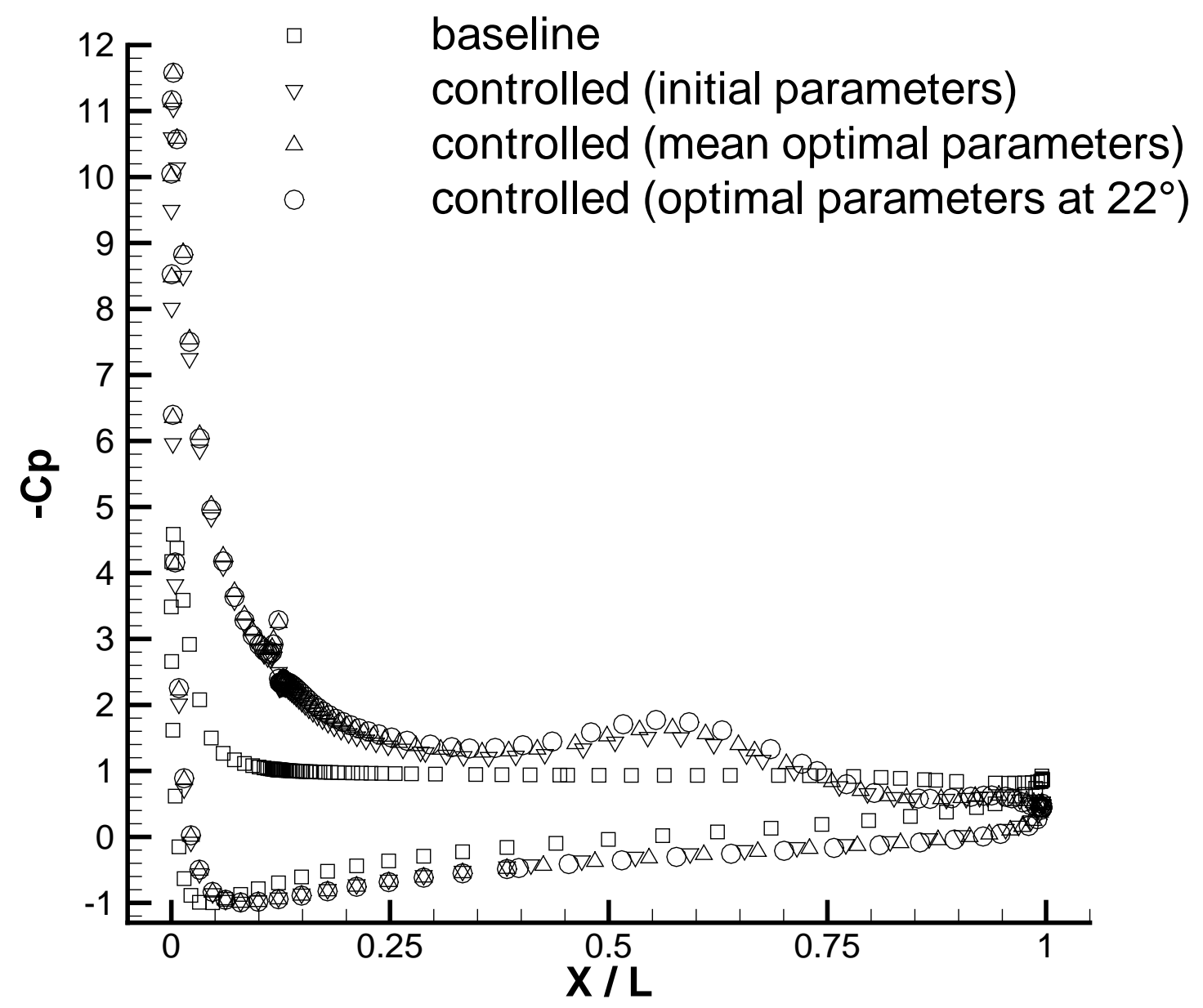

Figure 24: Time-averaged pressure coefficient at $22^{\circ}$ 


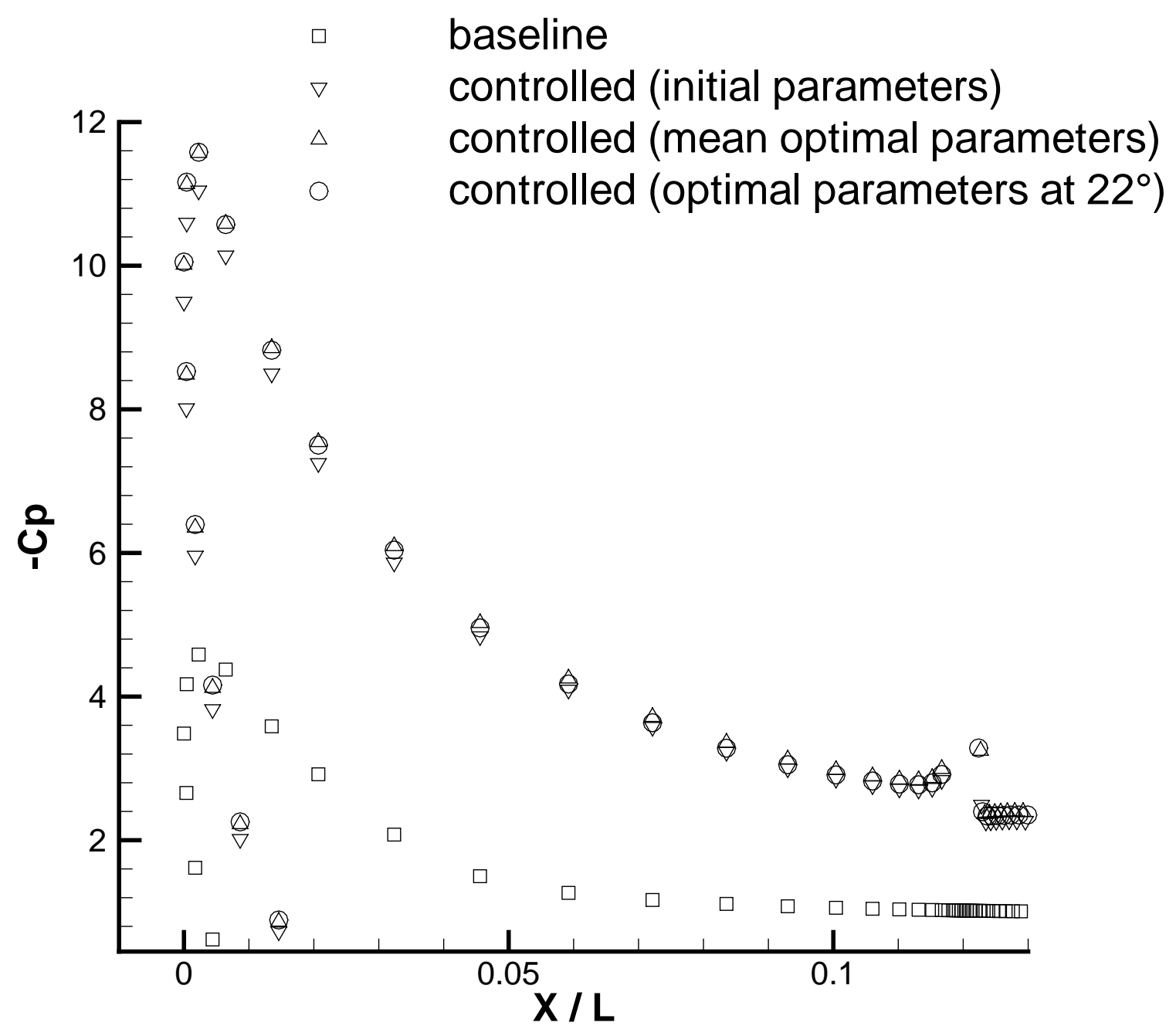

Figure 25: Time-averaged pressure coefficient at $22^{\circ}$ (zoom) 


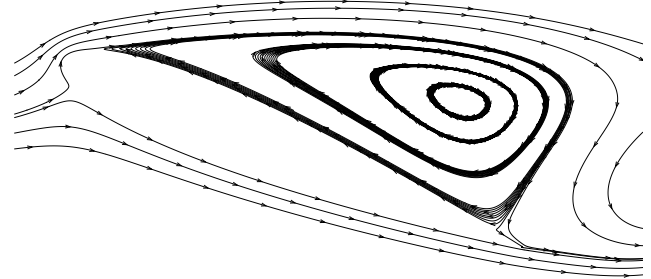

(a) $\Phi=0$

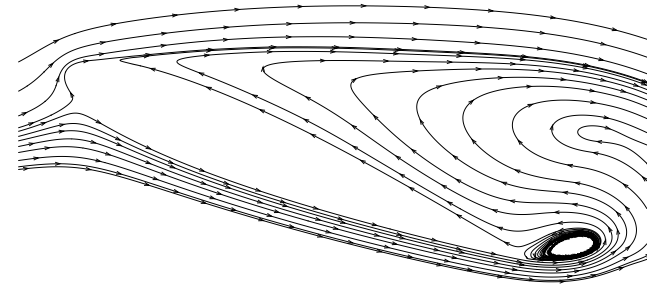

(c) $\Phi=\pi$

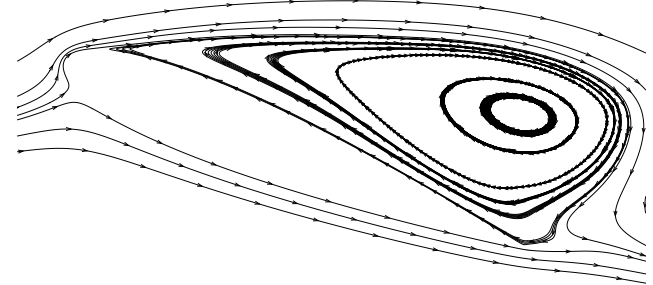

(b) $\Phi=\pi / 2$

Figure 26: Streamlines for baseline airfoil 

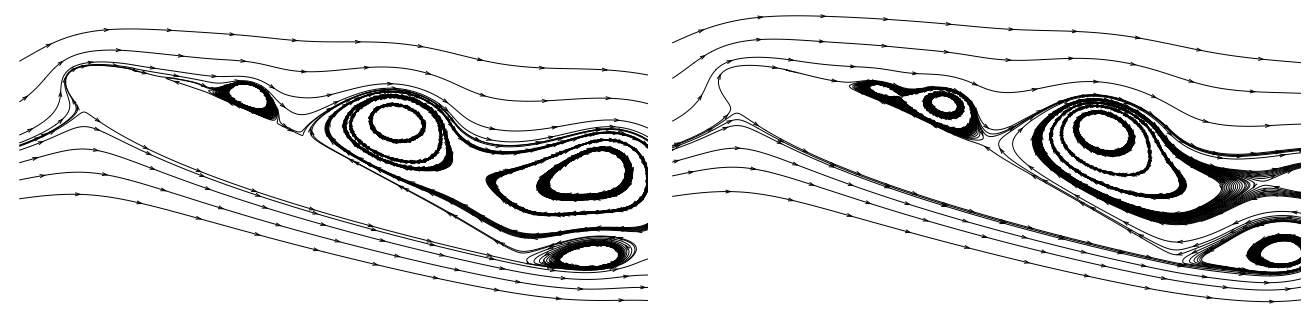

(a) $\Phi=0$

(b) $\Phi=\pi / 2$

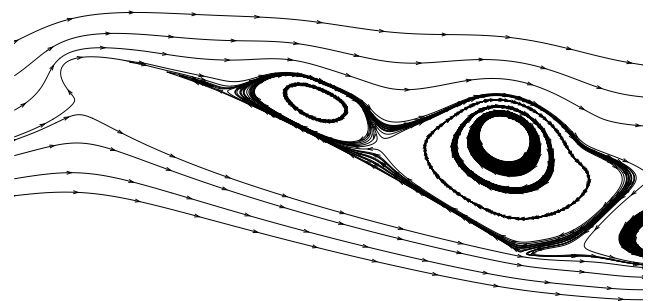

(c) $\Phi=\pi$

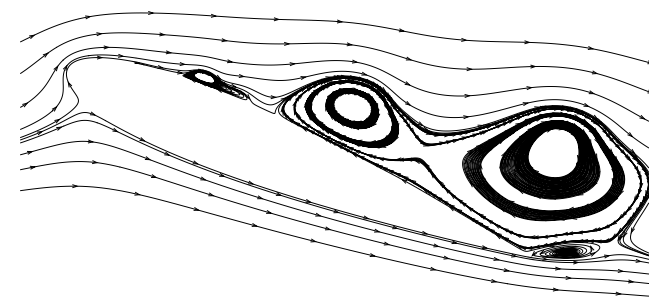

(d) $\Phi=3 \pi / 2$

Figure 27: Streamlines with initial parameters 


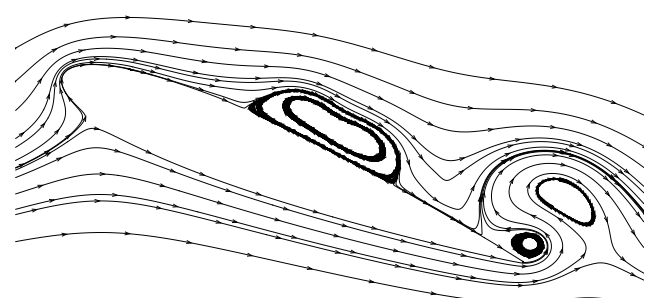

(a) $\Phi=0$

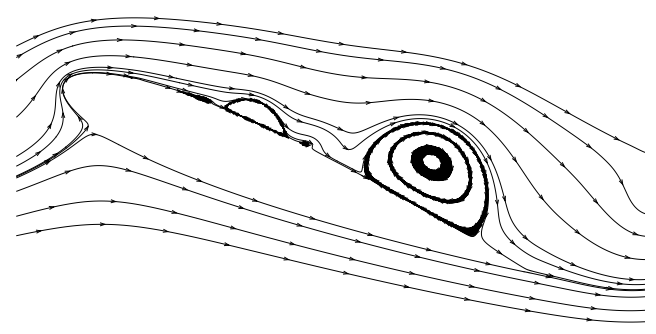

(c) $\Phi=\pi$

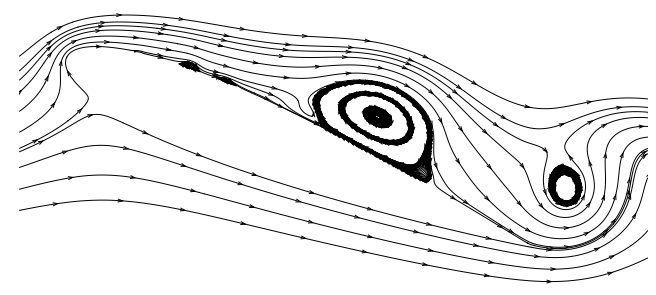

(b) $\Phi=\pi / 2$

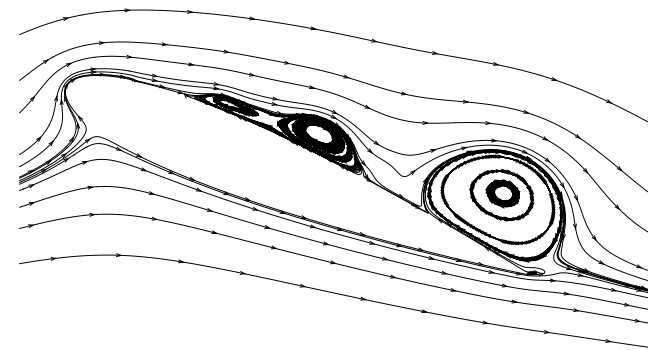

(d) $\Phi=3 \pi / 2$

Figure 28: Streamlines with mean optimal parameters 


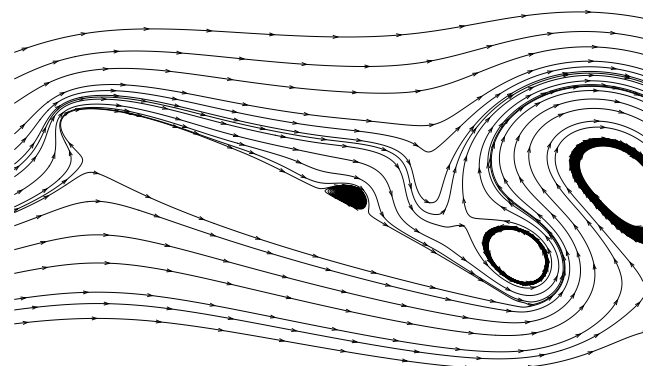

(a) $\Phi=0$

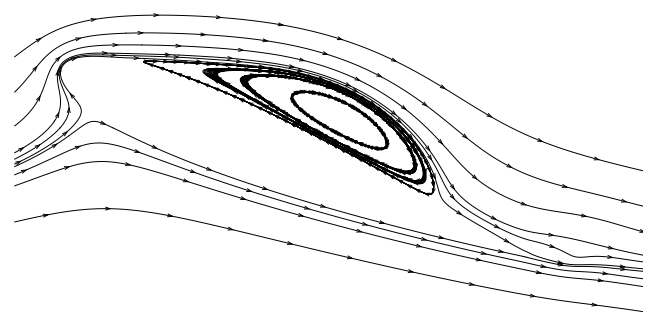

(c) $\Phi=\pi$

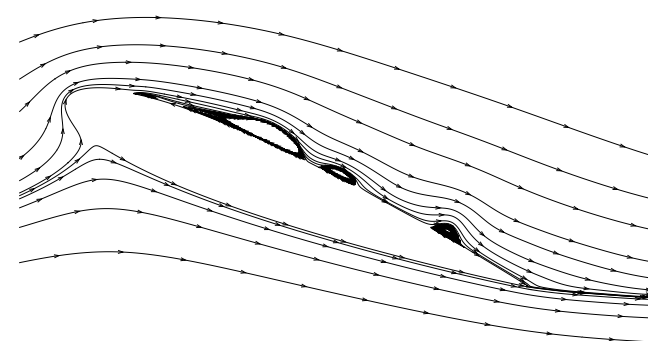

(b) $\Phi=\pi / 2$

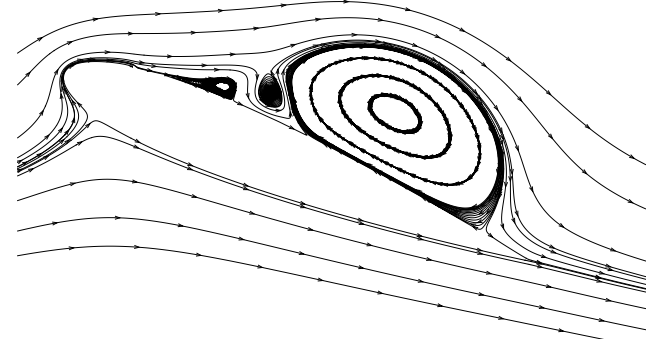

(d) $\Phi=3 \pi / 2$

Figure 29: Streamlines with optimal parameters at $22^{\circ}$ 